\title{
Transcriptional recording by CRISPR spacer acquisition from RNA
}

\author{
Florian Schmidt ${ }^{1}$, Mariia Y. Cherepkova ${ }^{1} \&$ Randall J. Platt ${ }^{1,2 *}$
}

\author{
${ }^{1}$ Department of Biosystems Science and Engineering, ETH Zurich, Basel, Switzerland. \\ ${ }^{2}$ Department of Chemistry, University of Basel, Basel, Switzerland. \\ *email: rplatt@ethz.ch
}

The ability to record transcriptional events within a cell over time would help to elucidate how molecular events give rise to complex cellular behaviours and states. However, current molecular recording technologies capture only a small set of defined stimuli. Here, we use CRISPR spacer acquisition to capture and convert intracellular RNAs into DNA, enabling DNA-based storage of transcriptional information. In Escherichia coli, we show that defined stimuli, such as an RNA virus or arbitrary sequences, as well as complex stimuli, such as oxidative stress, result in quantifiable transcriptional records that are stored within a population of cells. We demonstrate that the transcriptional records enable us to classify and describe complex cellular behaviours and to identify the precise genes that orchestrate differential cellular responses. In the future, CRISPR spacer acquisition-mediated recording of RNA followed by deep sequencing (Record-seq) could be used to reconstruct transcriptional histories and thereby that describe complex cell behaviours or pathological states.

A central challenge in biology is to understand how the molecular components of a cell function and integrate to enable complex cell behaviours. This challenge has fuelled the creation of increasingly sophisticated technologies that facilitate detailed intracellular observations at the levels of DNA, RNA, protein, and metabolites ${ }^{1}$. In particular, RNA sequencing technologies enable researchers to quantify transcriptomes within multiple or single cells, revealing the molecular signatures of cell behaviours, states, and types with unprecedented detail ${ }^{2,3}$. Despite the power of these technologies, they require destructive methods and therefore observations are limited to a few snapshots in time or to select asynchronous cellular processes. One provocative solution to this is to introduce synthetic memory devices ${ }^{4}$ within cells that enable the encoding, storage, and retrieval of transcriptional information. 
The bacterial adaptive immune system CRISPR-Cas embodies the ideal molecular recorder. Molecular memories of plasmid or viral infections are stored within CRISPR arrays in the form of short nucleic acid segments (spacers) separated by direct repeats. New memories are acquired via the action of a complex of Cas1 and Cas2, which integrates new spacers ahead of old spacers within the CRISPR array, thereby providing a temporal memory of molecular events $^{5-14}$. The prototype type I-E CRISPR acquisition system from E. coli was recently leveraged to store arbitrary information ${ }^{15,16}$ and quantifiable records of defined stimuli within bacterial populations ${ }^{17}$. These systems elegantly demonstrate the potential of using CRISPR spacer acquisition as a molecular recorder, but they are currently limited by the need to electroporate chemically synthesized nucleotides ${ }^{15,16}$ or, analogous to previous technologies, the availability of inducible promoters ${ }^{15-25}$. Moreover, these systems acquire spacers derived from DNA but not RNA, and therefore do not globally reflect the transcriptional history of a cell. While one naturally occurring fusion protein between Cas 1 and a reverse transcriptase (RT) domain (RT-Cas1) was recently experimentally validated to acquire spacers directly from RNA, it did not maintain this function heterologously in $E$. coli $^{26}$.

We hypothesized that direct CRISPR spacer acquisition from RNA could be leveraged to store transcriptional records in CRISPR arrays within living cells (Fig. 1a, b). Therefore, we characterized several orthologous RT-Cas1-containing CRISPR-Cas systems and found that one from Fusicatenibacter saccharivorans could acquire RNA spacers heterologously in E. coli. Leveraging F. saccharivorans RT-Cas1 and Cas2 (FsRT-Cas1-Cas2), we developed Recordseq, a method that enables transcriptome-scale molecular recordings into populations of cells. Transcriptional events are recorded according to RNA abundance, stored in CRISPR arrays within DNA, and can be used to describe both continuous and transient complex cellular behaviours.

\section{CRISPR spacer acquisition by FsRT-Cas1-Cas2}

We set out to identify an RT-Cas1-Cas2 CRISPR acquisition complex that could acquire spacers directly from RNA upon heterologous expression in E. coli. We identified 121 RT-Cas1 orthologues (Supplementary Table 1), and selected 14 of these for functional characterization (Extended Data Fig. 1a, b). We overexpressed corresponding RT-Cas 1 and Cas 2 proteins from a plasmid that also contained their predicted CRISPR array (Extended Data Fig. 1a, 
Supplementary Table 2). Using a previously established spacer acquisition assay ${ }^{27}$, we found that only one of the orthologues tested ( $F$. saccharivorans) actively acquired new spacers (Extended Data Fig. 1c). The endogenous $F$. saccharivorans locus contains two CRISPR arrays and we observed that novel spacers derived from the overexpression plasmid as well as the E. coli genome were acquired into either array (Extended Data Fig. 1c-e).

\section{Selective amplification of expanded CRISPR arrays}

Using the previously established spacer acquisition assay ${ }^{27}$, we obtained approximately 1,300 newly acquired spacers per 1 million deep sequencing reads for FsRT-Cas1-Cas2 (Extended Data Fig. 1c). To improve detection of novel spacers, we developed 'selective amplification of expanded CRISPR arrays' (SENECA), a method for selective amplification of CRISPR arrays that acquired new spacers (Fig. 2a, Extended Data Fig. 2a). A typical SENECA-assisted Recordseq experiment uses an input of about $180 \mathrm{ng}$ plasmid DNA extracted from an overnight culture of E. coli overexpressing FsRT-Cas1-Cas2, and yields 950,000 total spacers aligning to the plasmid or host genome for every 1 million sequencing reads (Fig. 2a, Extended Data Fig. 2b-e, Supplementary Notes). This represents an improvement of several thousand-fold compared to recent reports ${ }^{26,28}$. Using Record-seq, we readily demonstrated in vivo activity of FsRT-Cas1Cas2 in various E. coli strains and throughout growth phases (Extended Data Fig. 2b-g).

We then used Record-seq to rescreen our initial selection of RT-Cas1 orthologues (Extended Data Fig. 1b). Furthermore, we included all potential CRISPR arrays present in their endogenous loci in both possible directions in order to overcome the challenges associated with predicting these a priori (Extended Data Fig. 3a). Owing to the improved sensitivity of Recordseq compared to the classic readout, we readily detected newly acquired spacers for the majority of orthologues upon RT-Cas1-Cas2 expression (Extended Data Fig. 3b). Only a few orthologues exhibited a preferred directionality of the CRISPR array (that is, specificity for an upstream leader sequence). Consistent with the classic readout, FsRT-Cas1-Cas2 outperformed all other orthologues in terms of spacer acquisition efficiency and was chosen for further characterization. The concepts upon which Record-seq is based could also be used to characterize spacer acquisition in other CRISPR-Cas systems that have been intractable owing to low spacer acquisition efficiencies. 


\section{Characteristics of FsRT-Cas1-Cas2 spacer acquisition}

To better understand the properties of $F_{s} \mathrm{RT}-\mathrm{Cas} 1-\mathrm{Cas} 2$, we extensively characterized newly acquired spacers by performing Record-seq on populations of $E$. coli overexpressing FsRTCas1-Cas2 (Fig. 2a). We observed that genome-aligning spacers were preferentially acquired with a specific 'antisense' orientation, whereby spacers were complementary to the originating RNA (Fig. 2b, c). The median spacer length was 39 bp, with a distribution biased towards longer lengths (Fig. 2d). The median GC content was 36\%, showing a strong bias towards AT-rich spacers (Fig. 2e). In line with previously described type III CRISPR systems ${ }^{29}$, we did not find a sequence preference within or adjacent to newly adapted spacers acquired from either plasmid (Extended Data Fig. 4a) or genome (Fig. 2f), implying that the FsRT-Cas1-Cas2 complex has no protospacer adjacent motif (PAM). While observing spacer alignments to the E. coli genome, we noted that many coverage peaks were located near the termini of genes (Fig. 2b). Consistent with this observation, we found that at the genome-wide level, most spacers were derived from the $5^{\prime}$, and to a lesser extent, $3^{\prime}$ ends of genes (Fig. $2 \mathrm{~g}$ ). This finding raised the possibility that the apparent bias towards AT-rich spacers might be caused by the AT-richness of RNA ends in $E$. coli, but the bias towards AT-rich spacers persisted when considering only spacers derived from within the gene body (Extended Data Fig. 4b). We directly compared SENECA with the classic spacer readout to determine whether SENECA introduced additional biases, but found no major differences (Extended Data Fig. 4c-h). Together, these results reflect a process by which FsRTCas1-Cas2 selects AT-rich spacer-based sequences related to the beginning or end of a gene, such as the ends of an RNA molecule.

\section{FsRT-Cas1-Cas2 acquires spacers directly from RNA}

To determine whether FsRT-Cas1-Cas2 acquires spacers directly from RNA, we used a selfsplicing $t d$ group I intron ${ }^{30-32}$. This intron is a functional ribozyme that catalyses its own excision from the pre-mRNA, resulting in a characteristic splice junction that is not present at the DNA level. We constructed three intron-interrupted constructs based on genes that were highly sampled by spacers, namely $\operatorname{csp} A, \operatorname{rpoS}$ and $\operatorname{argR}$ (Fig. 3a). Upon expression of these constructs followed by Record-seq, we observed unique spacers spanning the splice junctions (Fig. 3a, b). To exclude the possibility that splice junction-containing spacers were acquired from extended complementary DNA copies generated through nonspecific reverse transcriptase activity in $E$. coli, we performed targeted deep sequencing on genomic DNA extracted from cultures 
expressing $t d$ intron constructs (Extended Data Fig. 5a), and found that the splice junction was absent at the DNA level (Extended Data Fig. 5a, b). Notably, these results do not exclude the possibility of spacer acquisition from DNA (Supplementary Notes). Thus, FsRT-Cas1-Cas2 facilitates CRISPR spacer acquisition from RNA heterologously in E. coli.

To further validate this finding, we used the Enterobacteria phage MS2. MS2 phages exist as both sense and antisense single-stranded RNAs during their lifecycle but have no DNA intermediates. Given that MS2 phages require the F pilus for cell entry, which is missing in $E$. coli BL21(DE3) cells, we turned to the $E$. coli K12 strain NovaBlue(DE3). Upon infection of FsRT-Cas1-Cas2-expressing cells with MS2 phage, we could readily observe novel MS2aligning spacers sampled from throughout the MS2 genome (Fig. 3c-e, Extended Data Fig. 5cf). The MS2-aligning spacers shared no sequence similarity with the plasmid or host genome, confirming their specificity (Extended Data Fig. 5d). In summary, FsRT-Cas 1-Cas2 enables spacer acquisition directly from a foreign RNA, thereby providing a molecular memory of an invading virus.

\section{Recording of arbitrary transcripts using Record-seq}

To assess the potential of Fs RT-Cas $1-$ Cas 2 for quantitatively recording transcriptional events, we used an inducible expression system to directly determine whether spacers were being acquired according to RNA abundance. The corresponding constructs contained super-folder GFP (sfGFP) or renilla luciferase (Rluc) genes under the transcriptional control of the anhydrotetracycline (aTc)-inducible $\mathrm{P}_{\text {teta }}$ promoter. We introduced these constructs into E coli cultured in increasing levels of aTc and subsequently collected both total RNA and plasmid DNA for qRT-PCR and Record-seq, respectively (Fig. 3f). Upon increasing induction of sfGFP or Rluc, there was a concordant dose-dependent increase in the coverage of spacers aligning to the respective coding sequence (Extended Data Fig. 6a). We quantified this response and observed a linear relationship $\left(R^{2}=0.97\right)$ between spacer counts and absolute mRNA copy number (Extended Data Fig. 6b-e) or aTc concentration in the medium (Fig. 3f). Furthermore, $s f G F P$-aligning spacers were readily detected against the backdrop of genome-aligning spacers by almost an order of magnitude (Extended Data Fig. 6f, g), which is in line with using a strong

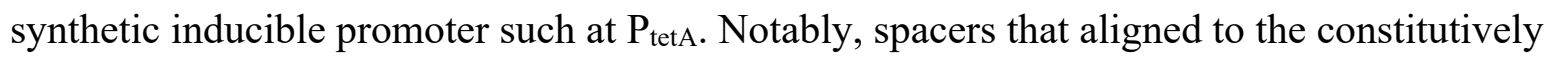
expressed KanR gene were not dependent on aTc concentration (Fig. 3f). 
To generalize these findings further, we evaluated a second inducible expression system, placing the firefly luciferase (Fluc) gene downstream of the 3-oxohexanoyl-homoserine lactone (3OC6-HSL)-inducible $P_{\text {LuxR }}$ promoter. Induction led to a fourfold increase in Fluc-aligning spacers (Extended Data Fig. 6h). Furthermore, combining both the aTc-inducible $\mathrm{P}_{\text {tetA }}$ and the 3OC6-HSL-inducible $\mathrm{P}_{\text {LuxR }}$ transcription system enabled orthogonal recording of two independent stimuli in parallel (Fig. 3g, Extended Data Fig. 6i, j). This suggests that Record-seq is compatible with seemingly any inducible expression system, thereby enabling recording of multiple orthogonal sets of defined stimuli within a population of living cells. Together, these results show that CRISPR spacer acquisition from RNA can generate a quantifiable record of cumulative transcript abundance, and also that the transcriptional records are efficiently retrieved using standard molecular and sequencing methods.

\section{Record-seq shows cumulatively highly expressed genes}

Considering that FsRT-Cas1-Cas2 acquired spacers directly from RNA in an abundancedependent manner, we investigated whether this could enable quantification of the cumulative cellular transcriptome. We collected both plasmid DNA for Record-seq and total RNA for RNAseq from cultures of E. coli overexpressing FsRT-Cas1-Cas2 (Fig. 4a). First, we confirmed the reproducibility of Record-seq between biological replicates (Pearson correlation, 0.996-0.999; $R^{2}=0.560-0.618$ ) (Extended Data Fig. 7a), and then we assessed the influence of gene expression on spacer acquisition. The FsRT-Cas1-Cas2 spacers showed a strong bias towards highly transcribed genes (Extended Data Fig. 7a) and correlated with RNA-seq-based gene expression values transcriptome-wide at various growth stages (Extended Data Fig. 7b-d). While certain CRISPR-Cas subtypes possess active mechanisms for preferentially acquiring plasmidderived spacers ${ }^{33}$, we did not observe the same after accounting for the high expression level of these genes (Extended Data Fig. 7e). Thus, spacers are systematically acquired from highly transcribed genes, and represent cumulative transcript expression.

\section{Transcriptome-scale recording reveals cell behaviours}

To determine whether Record-seq could be used to record and describe complex cellular behaviours, we turned to the well-studied oxidative stress and acid stress responses in E. coli. We performed Record-seq on oxidative and acid stress-stimulated ${ }^{34,35}$ cultures expressing FsRTCas1-Cas2 and analysed cumulative expression counts using unsupervised hierarchical 
clustering and principal component analysis (PCA). Both approaches were successful in distinguishing treatment conditions, suggesting that Record-seq captured the differential molecular histories (Fig. 4b-e). To identify the cumulatively differentially expressed genes, we leveraged standard differential expression analysis tools developed for RNA sequencing. To overcome specific biases and assumptions of individual tools, we used three complementary tools, namely DESeq $2^{36}$, edge ${ }^{37}$, and baySeq ${ }^{38}$. After identifying differentially expressed genes with each tool (Supplementary Table 9), we generated a set of signature genes for each stimulus based on the union of the top 20 differentially expressed genes from each analysis, which we hierarchically clustered and plotted along with their expression values (Fig. 4f, g). Among the signature genes, we identified several that were expected to dominate the cellular responses for each stimulus (Supplementary Notes). We investigated the minimum number of cells required for assessing complex cellular behaviours by Record-seq, finding that $8.8 \times 10^{6}$ cells are sufficient to appropriately classify treatment conditions (Extended Data Figs. 8, 9, Supplementary Notes). In sum, these data support the notion that the RNA-derived spacers stored within CRISPR arrays can be used to reconstruct the transcriptional response underlying a complex cellular behaviour.

\section{Sentinel cells encode transient herbicide exposure}

To determine whether Record-seq could be leveraged to produce sentinel cells, we used the herbicide paraquat and tested whether Record-seq could capture dose-dependent and transient exposure. Paraquat is a bacteriostatic herbicide that results in superoxide anion production in microbes ${ }^{39,40}$, and is banned in a number of countries owing to its acute toxicity in humans and use in suicide ${ }^{41}$.

Using an improved FsRT-Cas1-Cas2 expression construct (Extended Data Fig. 10a, b) we exposed $E$. coli cultures to increasing concentrations of paraquat and retrieved the transcriptional memories by Record-seq. Quantification of cumulative gene expression under the different treatment conditions showed that samples were readily classified into appropriate exposure groups using both unsupervised hierarchical clustering and PCA (Fig. 5a, b). Moreover, the signature genes captured dose-responsive and canonical paraquat-exposure genes within $E$. coli (Fig. 5c). For example, within the signature genes we found $a h p C$ and $a h p F$, which encode the two subunits of an alkyl hydroperoxide reductase previously shown to facilitate scavenging 
of reactive oxygen species (ROS) caused by paraquat ${ }^{42}$. In addition, we identified a set of genes of the cys-regulon involved in cysteine metabolism, namely cys $C$, cys $J$ and $c y s K$, which have been shown to facilitate paraquat resistance in $E$. coli ${ }^{42,43}$.

We next determined whether Record-seq could also capture transient paraquat exposure in a physiological range ${ }^{41}$. After transiently stimulating cultures with paraquat (Fig. $5 \mathrm{~d}$ ), we quantified cumulative gene expression and gene expression for Record-seq and RNA-seq data sets, respectively. Then, we assessed whether the two methods could capture the transient paraquat exposure using PCA (Fig. 5e, f) and differentially expressed signature gene clustering (Extended Data Fig. 10c, d). These analyses show that Record-seq, but not RNA-seq, was capable of capturing the transient paraquat exposure (Fig. 5e, f and Extended Data Fig. 10c, d). Together, these results show that the memory of paraquat exposure was lost within the cellular transcriptome as assessed by RNA-seq, but preserved within the molecular memories stored within the DNA of the CRISPR arrays of the sentinel cells, as investigated by Record-seq.

\section{Discussion}

Here, we have described Record-seq, a techique for encoding transcriptome-scale events into DNA and assessing the cumulative gene expression of populations of cells. We have demonstrated its potential by recording specific and complex transcriptional information. First, to improve upon existing spacer readout methods, we developed SENECA, resulting in a several thousand-fold improvement in spacer detection efficiency compared to recent reports ${ }^{26,44,45}$, thereby enabling in-depth characterization of FsRT-Cas1-Cas2 and its application as a molecular recorder. Our results suggest that RNA-derived spacers are preferentially acquired from the ends of abundant transcripts from AT-rich regions with no PAM, and are broadly sampled at the transcriptome-scale, enabling the parallelized quantification of cumulative transcript expression.

In a set of experiments, we have shown that upon increasing induction of arbitrary sequences, spacers are acquired in an orthogonal, dose-dependent manner and highly correlate with the absolute mRNA copy number in the cell, thus demonstrating that the molecular record faithfully recapitulates the initial stimulus in a predictable way. This also paves the way for increasingly multiplexed and orthogonal molecular recording devices. Upon inducing complex cellular behaviours, Record-seq provides a meaningful transcriptome-scale record of molecular 
events, which exceeds the capabilities of current molecular recording technologies that only record specific stimuli ${ }^{15-25}$. Finally, we used Record-seq to elucidate dose-dependent features of the complex cellular response to the bacteriostatic herbicide paraquat, and have shown that Record-seq, but not RNA-seq, can record transient paraquat stimulation.

Although additional work will greatly improve the capacity of Record-seq to encode richer and more dynamic expression and lineage information within fewer cells (Supplementary Notes), our proof-of-principle experiments introduce a powerful tool for recording transcriptome-scale events permanently in DNA for later reconstruction of complex molecular histories from populations of cells. The recorded transcriptional histories reflect the underlying gene expression changes and could therefore be used to interrogate biological or disease processes. In the long term, we envision that CRISPR spacer acquisition components could be introduced into other cell types to record the molecular sequences of events, and lineage paths, that gives rise to particular cell behaviours, cell states and types.

1. Karczewski, K. J. \& Snyder, M. P. Integrative omics for health and disease. Nat. Rev. Genet. 19, 299-310 (2018).

2. Wang, Z., Gerstein, M. \& Snyder, M. RNA-Seq: a revolutionary tool for transcriptomics. Nat. Rev. Genet. 10, 57-63 (2009).

3. Ozsolak, F. \& Milos, P. M. RNA sequencing: advances, challenges and opportunities. Nat. Rev. Genet. 12, 87-98 (2011).

4. Schmidt, F. \& Platt, R. J. Applications of CRISPR-Cas for synthetic biology and genetic recording. Current Opinion in Systems Biology 5, 9-15 (2017).

5. Barrangou, R. et al. CRISPR provides acquired resistance against viruses in prokaryotes. Science 315, 1709-1712 (2007).

6. Mojica, F. J., Díez-Villaseñor, C., García-Martínez, J. \& Soria, E. Intervening sequences of regularly spaced prokaryotic repeats derive from foreign genetic elements. J. Mol. Evol. 60, 174-182 (2005). 
7. Bolotin, A., Quinquis, B., Sorokin, A. \& Ehrlich, S. D. Clustered regularly interspaced short palindrome repeats (CRISPRs) have spacers of extrachromosomal origin. Microbiology 151, 2551-2561 (2005).

8. Pourcel, C., Salvignol, G. \& Vergnaud, G. CRISPR elements in Yersinia pestis acquire new repeats by preferential uptake of bacteriophage DNA, and provide additional tools for evolutionary studies. Microbiology 151, 653-663 (2005).

9. Garneau, J. E. et al. The CRISPR/Cas bacterial immune system cleaves bacteriophage and plasmid DNA. Nature 468, 67-71 (2010).

10. van der Oost, J., Westra, E. R., Jackson, R. N. \& Wiedenheft, B. Unravelling the structural and mechanistic basis of CRISPR-Cas systems. Nat. Rev. Microbiol. 12, 479492 (2014).

11. Marraffini, L. A. \& Sontheimer, E. J. CRISPR interference: RNA-directed adaptive immunity in bacteria and archaea. Nat. Rev. Genet. 11, 181-190 (2010).

12. Amitai, G. \& Sorek, R. CRISPR-Cas adaptation: insights into the mechanism of action. Nat. Rev. Microbiol. 14, 67-76 (2016).

13. Jinek, M. et al. A programmable dual-RNA-guided DNA endonuclease in adaptive bacterial immunity. Science 337, 816-821 (2012).

14. Sternberg, S. H., Richter, H., Charpentier, E. \& Qimron, U. Adaptation in CRISPR-Cas Systems. Mol. Cell 61, 797-808 (2016).

15. Shipman, S. L., Nivala, J., Macklis, J. D. \& Church, G. M. Molecular recordings by directed CRISPR spacer acquisition. Science 353, aaf1 175 (2016).

16. Shipman, S. L., Nivala, J., Macklis, J. D. \& Church, G. M. CRISPR-Cas encoding of a digital movie into the genomes of a population of living bacteria. Nature 547, 345-349 (2017).

17. Sheth, R. U., Yim, S. S., Wu, F. L. \& Wang, H. H. Multiplex recording of cellular events over time on CRISPR biological tape. Science 358, 1457-1461 (2017).

18. Perli, S. D., Cui, C. H. \& Lu, T. K. Continuous genetic recording with self-targeting CRISPR-Cas in human cells. Science 353, aag0511 (2016). 
19. Frieda, K. L. et al. Synthetic recording and in situ readout of lineage information in single cells. Nature 541, 107-111 (2017).

20. Tang, W. \& Liu, D. R. Rewritable multi-event analog recording in bacterial and mammalian cells. Science 360, eaap8992 (2018).

21. Farzadfard, F. \& Lu, T. K. Synthetic biology. Genomically encoded analog memory with precise in vivo DNA writing in living cell populations. Science 346, 1256272 , doi:10.1126/science.1256272 (2014).

22. McKenna, A. et al. Whole-organism lineage tracing by combinatorial and cumulative genome editing. Science 353, aaf7907 (2016).

23. Spanjaard, B. et al. Simultaneous lineage tracing and cell-type identification using CRISPR-Cas9-induced genetic scars. Nature biotechnology 36, 469-473, doi:10.1038/nbt.4124 (2018).

24. Kalhor, R., Mali, P. \& Church, G. M. Rapidly evolving homing CRISPR barcodes. Nat. Methods 14, 195-200 (2017).

25. Raj, B. et al. Simultaneous single-cell profiling of lineages and cell types in the vertebrate brain. Nat. Biotechnol. 36, 442-450 (2018).

26. Silas, S. et al. Direct CRISPR spacer acquisition from RNA by a natural reverse transcriptase-Cas1 fusion protein. Science 351, aad4234 (2016).

27. Yosef, I., Goren, M. G. \& Qimron, U. Proteins and DNA elements essential for the CRISPR adaptation process in Escherichia coli. Nucleic Acids Res. 40, 5569-5576 (2012).

28. Erdmann, S., Le Moine Bauer, S. \& Garrett, R. A. Inter-viral conflicts that exploit host CRISPR immune systems of Sulfolobus. Mol. Microbiol. 91, 900-917 (2014).

29. Pyenson, N. C., Gayvert, K., Varble, A., Elemento, O. \& Marraffini, L. A. Broad targeting specificity during bacterial type III CRISPR-Cas immunity constrains viral escape. Cell Host Microbe 22, 343-353.e3 (2017). 
30. Sandegren, L. \& Sjöberg, B.-M. Self-splicing of the bacteriophage T4 group I introns requires efficient translation of the pre-mRNA in vivo and correlates with the growth state of the infected bacterium. J. Bacteriol. 189, 980-990 (2007).

31. Belfort, M. et al. Processing of the intron-containing thymidylate synthase (td) gene of phage T4 is at the RNA level. Cell 41, 375-382 (1985).

32. Gott, J. M., Shub, D. A. \& Belfort, M. Multiple self-splicing introns in bacteriophage T4: evidence from autocatalytic GTP labeling of RNA in vitro. Cell 47, 81-87 (1986).

33. Levy, A. et al. CRISPR adaptation biases explain preference for acquisition of foreign DNA. Nature 520, 505-510 (2015).

34. Zheng, M. et al. DNA microarray-mediated transcriptional profiling of the Escherichia coli response to hydrogen peroxide. J. Bacteriol. 183, 4562-4570 (2001).

35. Maurer, L. M., Yohannes, E., Bondurant, S. S., Radmacher, M. \& Slonczewski, J. L. pH regulates genes for flagellar motility, catabolism, and oxidative stress in Escherichia coli K-12. J. Bacteriol. 187, 304-319 (2005).

36. Love, M. I., Huber, W. \& Anders, S. Moderated estimation of fold change and dispersion for RNA-seq data with DESeq2. Genome Biol. 15, 550 (2014).

37. Robinson, M. D., McCarthy, D. J. \& Smyth, G. K. edgeR: a Bioconductor package for differential expression analysis of digital gene expression data. Bioinformatics 26, 139140 (2010).

38. Hardcastle, T. J. \& Kelly, K. A. baySeq: empirical Bayesian methods for identifying differential expression in sequence count data. BMC Bioinformatics 11, 422 (2010).

39. Hassan, H. M. \& Fridovich, I. Paraquat and Escherichia coli. Mechanism of production of extracellular superoxide radical. J. Biol. Chem. 254, 10846-10852 (1979).

40. Ochsner, U. A., Vasil, M. L., Alsabbagh, E., Parvatiyar, K. \& Hassett, D. J. Role of the Pseudomonas aeruginosa oxyR-recG operon in oxidative stress defense and DNA repair: OxyR-dependent regulation of katB-ankB, ahpB, and ahpC-ahpF. J. Bacteriol. 182, 4533-4544 (2000). 
41. Wesseling, C., Corriols, M. \& Bravo, V. Acute pesticide poisoning and pesticide registration in Central America. Toxicol. Appl. Pharmacol. 207 (Suppl.), 697-705 (2005).

42. Pomposiello, P. J., Bennik, M. H. \& Demple, B. Genome-wide transcriptional profiling of the Escherichia coli responses to superoxide stress and sodium salicylate. J. Bacteriol. 183, 3890-3902 (2001).

43. Fuentes, D. E. et al. Cysteine metabolism-related genes and bacterial resistance to potassium tellurite. J. Bacteriol. 189, 8953-8960 (2007).

44. Silas, S. et al. Type III CRISPR-Cas systems can provide redundancy to counteract viral escape from type I systems. eLife 6, e27601 (2017).

45. Silas, S. et al. On the origin of reverse transcriptase - using CRISPR-Cas systems and their hyperdiverse, enigmatic spacer repertoires. MBio 8, e00897-17 (2017).

Acknowledgements We thank M. Okoniewski for assistance with data analysis; S. Ghosh and T. Tanna for technical assistance; S. Silas, A. Z. Fire, and the entire Platt Laboratory for discussions; S. Panke, M. Jeschek, L. Pestalozzi, I. Wüthrich, and D. Gerngross for reagents and comments; C. Beisel, E. Burcklen, K. Eschbach, I. Nissen, and M. Kohler from the Genomics Facility Basel for assistance in Illumina sequencing. R.J.P., M.Y.C and F.S. are supported, in part, by funds from the Swiss National Science Foundation, ETH domain Personalized Health and Related Technologies, Brain and Behavior Research Foundation, and the National Centres of Competence Molecular Systems Engineering.

Author contributions F.S. and M.Y.C, performed the experiments; F.S., M.Y.C, and R.J.P. analysed the data; and F.S., M.Y.C, and R.J.P. wrote the manuscript.

Correspondence and requests for materials should be addressed to R.J.P. 


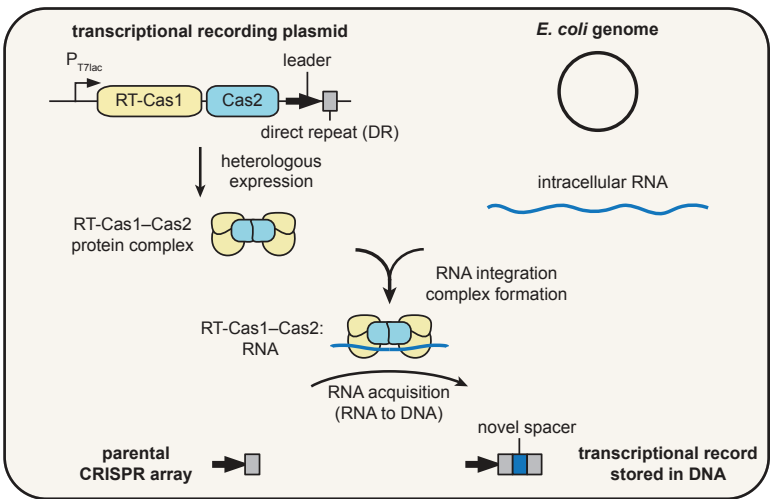

b

transcriptional snapshots versus transcriptional records

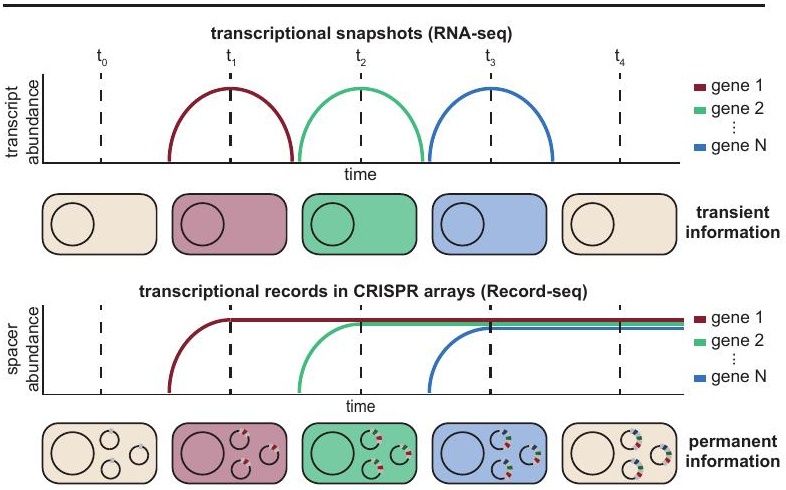


Fig. 1 | Transcriptional recording by CRISPR spacer acquisition from RNA. a, Expression of RT-Cas1-Cas2 leads to the acquisition of intracellular RNAs, providing a molecular memory of transcriptional events stored within DNA. b, Comparison of RNA-seq and Record-seq. RNAseq captures the transcriptome of a population of cells at a single point in time, providing a transient snapshot of cellular events. By contrast, Record-seq permanently stores information about prior transcriptional events in a CRISPR array, providing a molecular record that can be used to reconstruct transcriptional events that occurred over time. 
Fig. 2 Characterization of spacers acquired by FsRT-Cas1-Cas2. a, Schematic of Recordseq experimental workflow (Extended Data Fig. 2a). DR, direct repeat. b, Coverage of spacers aligning to the $E$. coli genome and a representative locus. Identical alignments represent recurrent spacers acquired in independent biological samples $(n=14)$. c, Spacers aligning to sense or antisense strand of coding genes in the E. coli genome. The sense/antisense orientation label in $\mathbf{c}, \mathbf{d}$ is with respect to the RNA $\mathbf{d}$, Length distribution of genome-aligning spacers. e, GC content distribution of genome-aligning spacers. Dotted line represents $50 \%$ GC content. f, Nucleotide probabilities of the 5' (left) or 3' (right) end of the spacer, along with the respective flanking sequence. Spacer (blue) and flanking (grey) nucleotides are shown. Data represent spacers merged across $n=14$ independent biological samples. g, Gene body coverage of spacer alignments along transcripts. Relative position represents percentiles of coding sequence lengths $\pm 300 \mathrm{bp}$ of adjacent genomic regions. Values are mean normalized coverage \pm s.d., $n=14$ independent biological samples. Values in $\mathbf{c}-\mathbf{e}$ are mean percent of genome-aligning spacers \pm s.e.m., $n=14$ independent biological samples with $\mathbf{c}$ showing individual data points. 
Figure 3 | FsRT-Cas1-Cas2 acquires spacers directly from RNA according to abundance.

a, Schematic of $t d$ intron-containing constructs and representative spacers aligning to the $t d$ intron splice junction. b, Quantification of spacers derived from the $t d$ intron splice junction. Values are mean \pm s.e.m. $t d$ intron spacers per million reads, $n=3$ independent biological samples. The sum of raw sequencing counts is shown below. c, Experimental workflow depicting MS2 recording. d, Quantification of MS2-derived RNA spacers. Values are mean \pm s.e.m. MS2-aligning spacers per million reads , $n=3$ (no MS2) and 4 (MS2) biologically independent samples. e, Coverage of spacers aligning to the MS2 genome. Data represent alignments merged across samples. Sense or antisense orientation is given with respect to the plus-strand MS2 RNA. f, Schematic and quantification of transcriptional recording of arbitrary sequences. Values are mean \pm s.e.m. relative spacer count, $n=10$ independent biological samples. The constitutively expressed $K a n R$ selection marker was used as a control. g, Schematic and quantification of orthogonal transcriptional recording. Values are mean \pm s.e.m. relative spacer count, $n=10$ (treated) and 9 (untreated) independent biological samples. 
a library prep \& deep sequencing

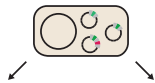

total RNA

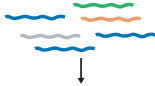

cDNA library plasmid DNA

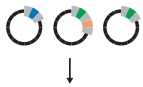

SENECA library alignment \& quantification

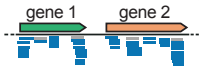

$\downarrow$ count table

\begin{tabular}{c|c|c} 
& sample A & sample B \\
\hline gene 1 & 3 & 9 \\
\hline gene 2 & 91 & 0 \\
\hline$\vdots$ & $\vdots$ & $\vdots$
\end{tabular}

analysis

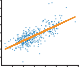

correlation

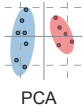

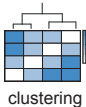

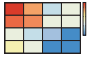

differential expression b

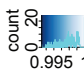
口oxidative stress untreated

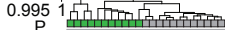

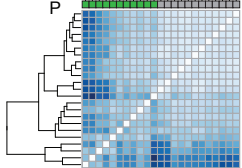

c

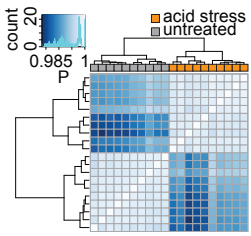

d

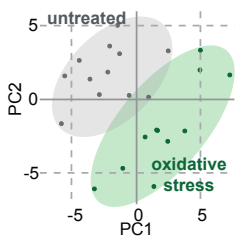

e

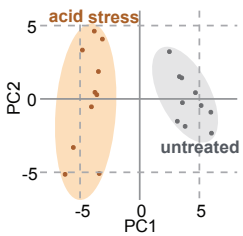

f

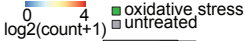

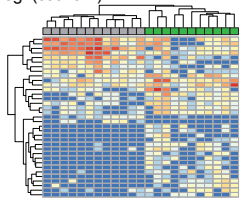

g
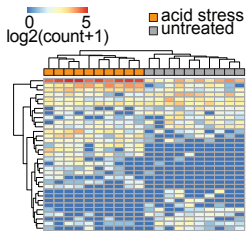
Figure 4 | Transcriptome-scale recording and analysis of complex cellular behaviours. a, Workflow for comparing Record-seq with RNA-seq. See Supplementary Notes for further discussion. b, Clustering of Record-seq data from untreated (grey) and oxidative-stress-treated (green) E. coli populations, performed using Pearson correlation, $n=12$ (untreated) and $n=11$ (treated) independent biological samples. c, Clustering of Record-seq data from untreated (grey boxes) and acid-stress-treated (orange boxes) E. coli populations, performed using Pearson correlation, $n=10$ independent biological samples. d, PCA of Record-seq data from untreated (grey) and oxidative-stress-treated (green) E. coli populations, $n=12$ (untreated) and $n=11$ (treated) independent biological samples. e, PCA of Record-seq data from untreated (grey) and acid-stress-treated (orange) E. coli populations, $n=10$ independent biological samples. $\mathbf{f}$, Clustering of Record-seq data for signature differentially expressed genes under oxidative stress. g, Clustering of Record-seq data for signature differentially expressed genes under acid stress. 
$10 \mathrm{mM} \square 1 \mathrm{mM}$ paraquat $\square$ unexposed

${ }^{0.6}{ }^{\prime}{ }^{1} 1$

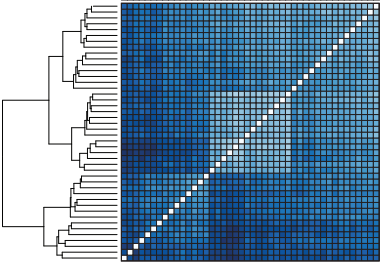

d

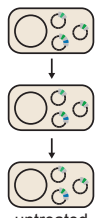

untreated
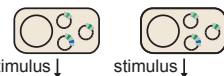

stimulus $\downarrow$
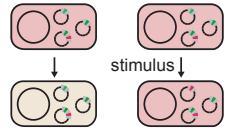

stimulus $\downarrow$

transient paraquat

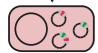

paraquat

000 plasmid DNA

Record-seq $\simeq \overline{\text { total RNA }}$

RNA-seq b

$\begin{array}{llll}0 & 2 & 4 & 6\end{array}$ $\log 2$ (count +1$)$

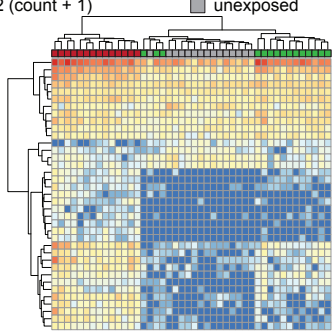

e

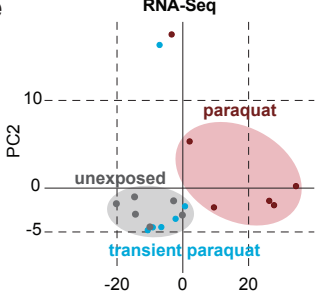

Record-Seq

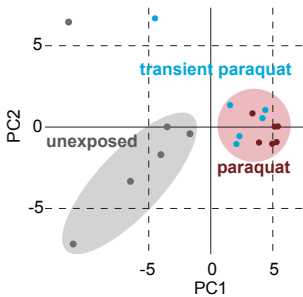


Figure 5 | Sentinel cells for recording of dose-dependent and transient herbicide exposure.

a, Clustering of Record-seq data from untreated (grey), $10 \mathrm{mM}$ paraquat-treated (red) and $1 \mathrm{mM}$ paraquat-treated (green) E. coli populations, performed using Pearson correlation, $n=15$ independent biological samples. b, PCA of Record-seq data from untreated (grey), $10 \mathrm{mM}$ paraquat-treated (red) and $1 \mathrm{mM}$ paraquat-treated (green) E. coli populations, $n=15$ independent biological samples. c, Clustering of Record-seq data for signature differentially expressed genes. d, Workflow for comparing Record-seq with RNA-seq for analysis of transient paraquat exposure. e, PCA of RNA-seq data from unexposed (grey), transiently paraquat-exposed (turquoise) and constantly paraquat-exposed (red) E. coli populations, $n=6$ independent biological samples. f, PCA of Record-seq data from unexposed (grey), transiently paraquatexposed (turquoise) and constantly paraquat-exposed (red) E. coli populations, $n=6$ independent biological samples. 


\section{METHODS}

\section{Orthologue discovery pipeline}

The protein sequence of Arthrospira platensis RT-Cas1 (WP_006620498) was used as a seed sequence, and a JACKHMMER search was run against all NCBI non-redundant protein sequences using HMMER v3.1b2 $\left(E \text {-value cutoff of } 1 \times 10^{-5}\right)^{46}$. Proteins with both Cas1 and reverse transcriptase domains were subsequently identified using HMMSCAN ( $E$-value cutoff of $\left.1 \times 10^{-5}\right)$. Genome sequence information for the candidate proteins was retrieved and further inspected for the presence of RT-Cas 1, Cas2, and a CRISPR array using CRISPRdetect v2.047, CRISPRone $^{48}$, and HMMSCAN ${ }^{46}$. From 121 candidate proteins, 14 CRISPR loci were selected and subsequently aligned using MUSCLE v3.8.31 ${ }^{49}$ to identify candidate domains and catalytic residues. Genetic distances were computed using the Jukes-Cantor method and a phylogenetic tree was built using the nearest-neighbour method.

\section{Bacterial strains and culture conditions}

E. coli strains used in this study were Stbl3 (Thermo Fisher Scientific) for cloning purposes, BL21(DE3) Gold (Agilent Technologies), BL21AI (Invitrogen) and NovaBlue(DE3) (EMD Millipore) as a K12 strain for acquisition assays. All strains were made competent using the Mix \& Go E. coli Transformation Kit \& Buffer Set (Zymo Research) following the manufacturer's protocol with growth in ZymoBroth at $19^{\circ} \mathrm{C}$ directly from fresh colonies. After transformation, cells were grown at $37^{\circ} \mathrm{C}$ on lysogenic broth (LB) (Difco) $1.5 \%$ agar plates containing $50 \mu \mathrm{g} / \mathrm{ml}$ kanamycin and $1 \%$ glucose $(\mathrm{w} / \mathrm{v})$ to reduce background expression from the T7lac system. Liquid cultures for plasmid isolation were grown in TB medium (24 g/l yeast extract, $20 \mathrm{~g} / 1$ tryptone, $4 \mathrm{ml} / 1$ glycerol, $17 \mathrm{mM} \mathrm{KH}_{2} \mathrm{PO}_{4}, 72 \mathrm{mM} \mathrm{K}_{2} \mathrm{HPO}_{4}$ ) containing 1\% glucose (w/v).

\section{Generation of Golden Gate-compatible pET30 overexpression vector}

All standard PCRs for cloning were performed using Phusion Flash High-Fidelity PCR Master Mix (Thermo Scientific) or KAPA HiFi HotStart ReadyMix (Roche), oligonucleotides and gBlocks were ordered from Integrated DNA technologies. Primers are listed in Supplementary Table 7. pET30b(+) (kind gift from M. Jeschek) was PCR amplified as five fragments using primers FS_151/FS_152, FS_153/FS_154, FS_155/FS_156, FS_157/FS_158, FS_159/FS_160, respectively, to remove the five undesired BbsI restriction sites present in the backbone. The resulting PCR fragments were assembled using $2 \times \mathrm{HiFi}$ DNA Assembly Mastermix (NEB), 
yielding pFS_0012. Subsequently, oligos FS_380 and FS_381 were annealed to generate a double-stranded DNA (dsDNA) fragment encoding the T7 terminator and cloned into pFS_0012 using XhoI/CsiI, yielding pFS_0013 - a pET30-derived overexpression vector harbouring two Golden Gate cloning sites and thus facilitating parallel cloning of RT-Cas1, Cas2 and a corresponding CRISPR array. Nucleotide sequences of all RT-Cas1 and Cas2 orthologues tested in this study along with their corresponding CRISPR arrays are listed under Supplementary Sequences.

\section{Golden Gate assembly of RT-Cas1-Cas2 overexpression vectors for orthologue screen}

RT-Cas1, Cas2 and CRISPR array sequences were ordered from Twist Biosciences and Genscript. Putative CRISPR arrays were ordered as sequences consisting of the leader sequence followed by DR-nativespacer1-DR-nativespacer2-DR. Furthermore, each fragment was flanked by BbsI restriction sites generating overhangs facilitating Golden Gate Assembly into pFS_0013. In brief, 40 fmol per fragment (RT-Cas1, Cas2, corresponding CRISPR array, pFS_0013 acceptor vector), $1 \mu \mathrm{ATP} / \mathrm{DTT} \operatorname{mix}$ (10 mM each), $0.25 \mu 1$ T7 DNA Ligase (Enzymatics), 0.75 $\mu 1$ BpiI (Thermo Scientific), $1 \mu 1$ buffer green up to $10 \mu 1$ with PCR grade $\mathrm{H}_{2} \mathrm{O}$ were subjected to 99 cycles of $37{ }^{\circ} \mathrm{C}$ for $3 \mathrm{~min}, 16^{\circ} \mathrm{C}$ for $5 \mathrm{~min}$, followed by $80^{\circ} \mathrm{C}$ for $10 \mathrm{~min}$. Subsequently, $5 \mu 1$ of this mixture was transformed into $50 \mu \mathrm{S} \mathrm{Stbl3}$ cells and recovered in SOC medium for $30 \mathrm{~min}$ at $37^{\circ} \mathrm{C}, 1,000 \mathrm{rpm}$ before spreading on plates.

\section{Spacer acquisition}

Acquisition assays were performed at $37^{\circ} \mathrm{C}, 300$ r.p.m. in bacterial culture tubes containing $3 \mathrm{ml}$ TB medium supplied with $100 \mu \mathrm{M}$ isopropyl- $\beta$-D-thiogalactopyranoside (IPTG) (Sigma Aldrich) and for BL21(DE3) Gold and NovaBlue(DE3). For E. coli BL21AI, L-(+)-arabinose (Sigma Aldrich) was additionally added to $0.2 \%(\mathrm{w} / \mathrm{v})$. Each culture was inoculated with two colonies of bacteria stored no longer than 14 days at $4{ }^{\circ} \mathrm{C}$ upon transformation and overnight growth at 37 ${ }^{\circ} \mathrm{C}$. When cultures reached saturation (typically $12-14 \mathrm{~h}$ after inoculation), $2 \mathrm{ml}$ of bacterial culture was harvested and plasmids containing CRISPR arrays were isolated by standard plasmid Mini-Prep procedures to serve as a template for preparation of deep sequencing libraries.

\section{Amplification of CRISPR arrays for classical acquisition readout by deep sequencing}

Leader proximal spacers were PCR amplified from 3 ng plasmid DNA per $\mu 1$ PCR reaction using NEBNext High-Fidelity $2 \times$ PCR Master Mix (NEB) with a forward primer binding in the leader 
sequence of the respective CRISPR array and a reverse primer binding in the first native spacer (see Supplementary Informatinon (Primer Design Note 1) and Supplementary Table 3 for primer design and binding sites of individual CRISPR arrays, respectively). For each biological replicate, 12 individual PCR reactions of $10 \mu 1$ were performed with an extension time of $15 \mathrm{~s}$ for 16 cycles. The individual $10-\mu 1$ reactions belonging to the same biological sample were then pooled, and residual primers removed using homemade AMPure beads at a PCR to bead ratio of 1:1.5 (v/v) eluting the PCR product in $60 \mu 1$ of buffer TE. Subsequently, $500 \mathrm{ng}$ of first round PCR product per biological sample was run on a 3\% Laboratory agarose gel (300V, 55 min, cooling the gel-chamber in an ice-water bath during the run) and purified by blind excision of gel slices at 211 to $300 \mathrm{bp}$, avoiding the prominent DNA band corresponding to PCR products of the unexpanded array (that is, no acquisition of novel spacers). Amplicons were then purified from the gel slices using the QIAquick Gel Extraction Kit (QIAGEN) and eluted into $22 \mu 1$ buffer EB. Illumina sequencing adaptors and indices were appended in a second round of PCR, using $6 \mu 1$ gel-purified input DNA as a template in a $20-\mu 1$ PCR reaction with universal second round deep sequencing primers attaching P5 and P7 handles for binding of PCR products to the flow cell in deep sequencing as well as barcoding the samples with $(N)_{8}$ barcodes corresponding to Illumina TruSeq HT indices (Supplementary Information (Primer Design Note 2) and Supplementary Table 4 for primer design and indices, respectively). After this second round of PCR, products were purified using the QIAquick PCR Purification Kit (QIAGEN) and eluted in $22 \mu \mathrm{l}$ buffer

EB. Samples were then pooled and subjected to another round of gel purification using the same parameters as described above, this time excising products in the range of 280 to $350 \mathrm{bp}$.

\section{Selective amplification of ExpaNdEd CRISPR arrays (SENECA)}

FsCRISPRArray2 was amplified from pFS_160 using FS_871/FS_904, generating a minimal Fs CRISPR array consisting of the leader sequence and a single direct repeat followed by a FaqI restriction site (CTTCAG) on the bottom strand resulting in plasmid pFS_0235 as our standard recording plasmid. This plasmid was transformed into chemocompetent BL21(DE3) Gold bacteria or NovaBlue(DE3) (EMD Millipore) and subjected to spacer acquisition as described above. Following plasmid extraction and quantification using Quant-IT PicoGreen dsDNA Assay Kit (Thermo Scientific) read out with a Tecan M1000 Pro Microplate reader, plasmid DNA was subjected to SENECA-adaptor ligation in a Golden Gate reaction. Oligonucleotides FS_0963/FS_0964 were annealed $(2.5 \mu \mathrm{l}$ each of $100 \mu \mathrm{M}$ oligo, $5 \mu \mathrm{l}$ NEBuffer 2 (NEB), $40 \mu 1$ 
PCR grade $\mathrm{H}_{2} \mathrm{O}$ ), by heating to $95^{\circ} \mathrm{C}$ for $5 \mathrm{~min}$ and cooling to $20^{\circ} \mathrm{C}$ at $0.12{ }^{\circ} \mathrm{C}$ per s. Annealed oligos were diluted 1:100 in TE buffer. Next, 40 fmol plasmid DNA (180.3 ng for pFS_0235), $0.25 \mu 1$ T7 ligase (Enzymatics), $1 \mu 1$ FastDigest FaqI $0.5 \mu 1$ of $20 \times \mathrm{SAM}, 1 \mathrm{mM}$ ATP, $1 \mathrm{mM}$ DTT (all Thermo Scientific), $1 \mu$ l of annealed, diluted oligonucleotides FS_0963/FS_0964 in 10 $\mu 1$ total volume were subjected to 99 cycles of $3 \min 37^{\circ} \mathrm{C}, 3 \min 20{ }^{\circ} \mathrm{C}$ followed by $15 \min$ at $55^{\circ} \mathrm{C}$. First-round deep sequencing PCR was performed using NEBNext High-Fidelity $2 \times$ PCR Master Mix (NEB) (forward primers: FS_0968 to FS_0974, reverse primer: FS_0911). For each biosample, one $30-\mu 1$ reaction containing $10.38 \mu 1$ adaptor ligated plasmid DNA was performed $\left(98^{\circ} \mathrm{C}\right.$ for $30 \mathrm{~s} ; 22$ cycles at $98^{\circ} \mathrm{C}$ for $10 \mathrm{~s}, 57^{\circ} \mathrm{C}$ for $30 \mathrm{~s}$ and $72{ }^{\circ} \mathrm{C}$ for $20 \mathrm{~s}$ followed by $72{ }^{\circ} \mathrm{C}$ for $5 \mathrm{~min}$ ), pooled and purified using magnetic beads (GE Healthcare) at a PCR-to-bead ratio of 1:1.6 (v/v) recovering the PCR product in $25 \mu 1 \mathrm{TE}$ buffer (Supplementary Information (Primer Design Note 3) for details on primer design). Illumina sequencing adaptors and indices were appended in a second round of PCR $\left(98^{\circ} \mathrm{C}\right.$ for $30 \mathrm{~s}, 8$ cycles of $98^{\circ} \mathrm{C}$ for $10 \mathrm{~s}, 65^{\circ} \mathrm{C}$ for $30 \mathrm{~s}$ and $72{ }^{\circ} \mathrm{C}$ for $30 \mathrm{~s}$, and $72{ }^{\circ} \mathrm{C}$ for $5 \mathrm{~min}$ ) using $5 \mu$ first-round PCR product as input in a $20-\mu 1$ reaction (Supplementary Information (Primer Design Note 2) and Supplementary Table 4 for primer design and indices, respectively). Samples were pooled, desalted using the QIAquick PCR Purification Kit (QIAGEN) and size selected on a E-Gel EX Agarose Gels, 2\% (Thermo Scientific), loading 200-500 ng DNA per lane, extracted using the QIAquick Gel Extraction Kit and subjected to deep sequencing on Illumina MiSeq or NextSeq500 platforms using the MiSeq Reagent Kit v3 (150 cycles) or NextSeq 500/550 Mid/High Output v2 kit (150 cycles) (both Ilumina), respectively. Libraries were loaded at a concentration of 1.4 to $1.6 \mathrm{pM}$ as determined by qPCR using the KAPA Library Quantification Kit for Illumina Platforms (Roche). PhiX was included at $5-10 \%$.

\section{SENECA-based orthologue screen}

For the SENECA-based CRISPR array directionality screen, putative CRISPR arrays were extracted from genomic sequences, assuming a standard leader length of $150 \mathrm{nt}$ followed by a single direct repeat. The FaqI restriction site required for SENECA was appended downstream of the direct repeat and sequences were flanked by universal adapters for amplification and cloning. The final array sequences including these features are provided in the Supplementary Information (Supplementary Sequences 2) and were ordered from Twist Biosciences as linear DNA fragments. These were PCR amplified using primers FS_1406/FS_1407 and cloned into 
Csil/NotI-digested plasmids containing their respective RT-Cas 1-Cas2 orthologue using HiFi DNA Assembly (NEB). Upon transformation into E. coli BL21(DE3), these constructs were subjected to the standard spacer acquisition assay in TB media. Plasmid DNA was extracted and subjected to SENECA adaptor ligation. The respective oligos to be annealed for each CRISPR array tested in this experiment are listed in Supplementary Table 5. Following adaptor ligation, a single $140 \mu \mathrm{l}$ first-round PCR reaction was prepared for each orthologue using NEBNext HighFidelity $2 \times$ PCR Master Mix and containing the entire 20- $\mu$ l SENECA adaptor ligation as a template. First-round PCR primers specific to the respective direct repeat of each CRISPR array tested are listed in Supplementary Table 6. The 140- $\mu$ l PCR reaction was split into 12 reactions of $11 \mu \mathrm{l}$ along the row of a 96-well plate. This plate was subjected to a gradient PCR (53 to $68^{\circ} \mathrm{C}$ in an Eppendorf Mastercycler Gradient). This procedure was chosen because SENECA leverages the fact that a direct repeat matching primer will only bind to the full direct repeat resulting from an acquisition event but not the truncated parental direct repeat at a unique annealing temperature. By splitting the PCR reaction and subjecting it to a temperature gradient, it is ensured that without a prior knowledge, at least one of the 12 reactions is subjected to the annealing temperature at which selective amplification of expanded CRISPR arrays occurs. PCR was performed for 30 cycles upon which, the 12 reactions performed along the temperature gradient were pooled again and purified using $1.85 \times$ Ampure beads and eluted in $25 \mu 1 \mathrm{TE}$ buffer. Five $\mu 1$ of this elution was used as a template for a standard $20-\mu 1$ second-round PCR at $65^{\circ} \mathrm{C}$ annealing temperature for 12 cycles as described above. Subsequently, PCR products were purified using $2.2 \times$ Ampure beads, eluted into $22 \mu 1 \mathrm{TE}$ buffer, size selected as described in the standard SENECA protocol (E-Gel Ex 2\%, followed by gel extraction) and subjected to deep sequencing.

\section{Deep sequencing}

Small-scale targeted deep sequencing of CRISPR arrays for the orthologue screen was performed using the Illumina MiSeq v3 300 cycle kit on an Illumina MiSeq platform or Illumina HiSeq High Output High Output PE 200 cycle kit an Illumina HighSeq2500. Deep sequencing of spacer libraries prepared using SENECA were sequenced using the NextSeq 550/550 High Output Kit v2 150 cycle on Illumina NextSeq platform or the MiSeq Reagent Kit v3 150-cycle on a MiSeq. 


\section{Data analysis pipeline}

FASTQ files were quality filtered and trimmed using trimmomatic (trimmomatic s.e.

LEADING:3 TRAILING:3 SLIDINGWINDOW:4:15 MINLEN:75) ${ }^{50}$ and subsequently converted to FASTA files using FASTX-Toolkit v0.0.14 (fastq-to-fasta)

(http://hannonlab.cshl.edu/fastx_toolkit/). Using custom scripts written in python2.7, spacers were identified based on the identification of a 20-66 nucleotide sequence between two 10-nt direct repeat segments, allowing for 2 and 3 mismatches in the first and second direct repeat segments, respectively. Arrays with multiple spacers were identified based on the presence of a complete direct repeat sequence, allowing for 3 mismatches. Only unique spacers ( $>1$ mismatch) from a given sample were further processed. Spacers were aligned to a merged reference genome containing plasmid and E. coli sequences (E. coli B121(DE3) Gold (NC_012947.1) genome, E. coli K12 (NC_000913.3)) using bowtie2 (bowtie2-very-sensitive-local) ${ }^{51}$. In MS2 challenge experiments, the MS2 sequence (MS2 (NC_001417.2)) was also included in the merged reference genome. Identical alignments were collapsed using samtoolsv1.352, and alignments were visualized in Geneiousv10.2.3. Basic statistics about numbers of reads or alignment features were calculated using standard bash commands, and compiled and visualized using Prism7.0d. Gene body percentiles were calculated using RSeQC (geneBody_coverage.py v2.6.4 $)^{53}$. Nucleotide probabilities were determined and visualized using the weblogo webtool v2.8.2 $2^{54}$. Simulated spacer data sets were prepared using BEDtools v2.25 (bedtools random -n $500-138)^{55}$. Transcript quantification for RNA-seq and Record-seq was performed using featureCounts v1.5.0 ${ }^{56}$. Using custom scripts written in Matlab v9.1.0, RNA-seq and Record-seq transcript counts were normalized using transcripts per million (TPM) and used to compute cumulative spacer sums, a linear regression fit, coefficient of determination $\left(R^{2}\right)$, and Pearson linear correlation coefficient.

Record-seq data sets corresponding to oxidative or acid stress treatments were analysed using custom scripts written in R v3.4.4. In brief, transcripts with fewer than 5 counts across replicates were discarded. Heatmaps representing unsupervised hierarchical clustering of Pearson linear correlation with complete linkage (using raw transcript counts as inputs) were prepared using the 'heatmap.2', 'hclust', and 'cor' commands with default settings. PCA was performed on $\log _{2}$ transformed data (raw counts plus one pseudocount to tolerate zeros) for the 50 most variable (s.d.) genes using the 'prcomp' command with default settings. Differential expression 
analyses (using raw counts plus one pseudocount as input) were performed using DEseq2v1.14.1, edgeRv3.16.5, and baySeqv2.8.0 encapsulations within R. Heatmaps representing unsupervised hierarchical clustering of signature differentially expressed genes were prepared using the 'pheatmap' command with default settings.

\section{RNASeq of E. coli BL21(DE3)}

RNA extraction from E. coli BL21(DE3) was performed after overnight growth under induction of FsRT-Cas1-Cas2 expression following the QIAGEN Supplementary Protocol: Purification of total RNA from bacteria using the RNeasy Mini Kit. To achieve the appropriate amount of input culture (corresponding to $5 \times 10^{8}$ cells), serial dilutions of the overnight culture were prepared to achieve an $\mathrm{OD}_{600}$ between 0.2 to 0.6 measured with a NanoDrop OneC (Thermo Scientific). Bacteria were lysed using acid-washed glass beads (G1277-10G, Sigma Aldrich). The additional on-column DNase digestion was performed using the RNase-Free DNase Set (QIAGEN). DNA free RNA was submitted to the Genomics Facility Basel for ribosomal RNA (rRNA) depletion using the Ribo-Zero rRNA Removal Kit (Illumina) and followed by library preparation and sequencing on an Illumina NextSeq platform using the NextSeq 500/550 High Output v2 kit (150 cycles).

\section{td intron}

The gBlock FS_gBlock_td_intron_acceptor (Supplementary Information, Sequences 3) was cloned into pFS_0235 using SphI/SgrAI yielding pFS_0238. This gBlock encoded the BBa_J23104 promoter, the ribosome binding site from bacteriophage T7 gene 10 as well as the $t d$ intron sequence including flanking regions facilitating efficient splicing. Furthermore, a BbsImediated Golden Gate cloning site was placed downstream and upstream of the $t d$ intron sequence, allowing for seamless assembly of upstream and downstream exon sequences in a single one-pot reaction as described above. As we previously noticed, that the $5^{\prime}$ end of transcripts was preferentially acquired by the FsRT-Cas1-Cas 2 complex, we introduced the $t d$ intron within the first 23 to 31 nucleotides of the respective transcripts. We created introninterrupted sequences of three $E$. coli genes $\operatorname{csp} A, \operatorname{rpoS}$ and $\arg R$ (which encode cold shock protein CspA, RNA polymerase sigma factor RpoS and arginine repressor, respectively). These were selected based on the fact that they were well sampled by the FsRT-Cas1-Cas 2 complex in preceding SENECA experiments. The flanking exon sequences were mutated in four to six 
positions to yield optimized sequences for $t d$ intron splicing, which also aided in unambiguously distinguishing the spliced and endogenous transcripts or DNA.

Accordingly, we ordered complementary oligonucleotides for the fragment of the transcript to be cloned $5^{\prime}$ of the $t d$ intron and annealed them before Golden Gate Assembly, while the fragment to be cloned 3' of the intron was amplified by PCR from genomic DNA. Oligonucleotides were FS_1054/1055 (5' of the intron, annealed) and FS_1056/1057 (3' of the intron, PCR) for CspA; FS_1038/1039 and FS_1040/1041 for RpoS; FS_1046/1047 and FS_1048/1049 for ArgR. We ensured that mutating sequences of the respective genes to those of the $t d$ intron flanking sites did not generate a stop codon. The $t d$ intron containing Fs RT-Cas1Cas2 overexpression constructs were subjected to a standard acquisition assay followed by plasmid DNA extraction, SENECA and deep sequencing. The presence of $t d$ intron splice sites in DNA outside of the FsCRISPR array was tested by extracting gDNA from $t d$-ArgR transformed cultures using the GenElute Bacterial Genomic DNA Kit (Sigma Aldrich). Libraries containing the $t d$ intron insertion site were amplified using a two-round PCR strategy method analogous to the ones described above using forward primers FS_1154 to FS_1157 and reverse primers FS_1158 to FS_1161 (Supplementary Table 7). First-round PCR was performed at $57{ }^{\circ} \mathrm{C}$ annealing temperature and $20 \mathrm{~s}$ elongation for 15 cycles. Second-round PCR was performed at $63{ }^{\circ} \mathrm{C}$ annealing temperature and 20 s elongation for 8 cycles.

\section{Infection with MS2 phage}

For infections with MS2 phage, the recording plasmid pFS_0235 was transformed into $\mathrm{F}^{\prime}$, and thus MS2-susceptible, NovaBlue(DE3) competent cells (EMD Millipore). Next morning, ten colonies were inoculated with $15 \mathrm{ml}$ TB containing $100 \mu \mathrm{M}$ IPTG and grown at $37^{\circ} \mathrm{C}, 150 \mathrm{rpm}$ in an orbital shaker until an $\mathrm{OD}_{600}$ of 0.24 . Then, $\mathrm{MgSO}_{4}$ was added to $5 \mathrm{mM}$ final concentration. Aliquots of $3 \mathrm{ml}$ were split into bacterial culture tubes, infected with $200 \mu$ high-titre MS2 phage suspension and incubated for $1 \mathrm{~h}$ at room temperature without shaking to allow infection by MS2. Next, culture tubes were transferred to the orbital shaker and incubated overnight at 30 ${ }^{\circ} \mathrm{C}, 80$ r.p.m. Growth of $E$. coli in presence of MS2 phage at $30^{\circ} \mathrm{C}$ rather than $37^{\circ} \mathrm{C}$ prevents lysis of cells by productive MS2. Next morning, shaking was increased to 150 r.p.m. Another day later ( $\sim 41 \mathrm{~h}$ post-infection), cultures were pelleted by centrifugation, and plasmid DNA was extracted and subjected to SENECA followed by deep sequencing. 


\section{Synthetic recording of sfGFP and Rluc transcripts}

The Pcat-tetR-term_PtetO encoding fragment was amplified with primers FS_1123/FS_1125 from pLP167 (kind gift from L. Pestalozzi), digested with BamHI/AgeI and cloned into AgeI/BbsI-digested pFS_0238 (see cloning of $t d$ intron constructs), yielding pFS_0270 which contains a BbsI-mediated Golden-Gate immediately downstream of the PtetA promoter. Subsequently, sfGFP was amplified from pLP167 with primers FS_1134/FS_1135 and Rluc was amplified using FS_1136/FS_1137 from BBa_J52008 (registry of standard biological parts). Both fragments were cloned into pFS_0270 using BbsI-mediated Golden Gate assembly ${ }^{57}$, yielding pFS_0271 (sfGFP) and pFS_0272 (Rluc). LuxR promoter parts were amplified with primers FS_1584/FS_1585 from pIG0046 and FS_1586/ FS_1587 from pIG0059 (registry of standard biological parts) and cloned into AgeI-digested pFS_0270 using NEBuilder HiFi DNA Assembly Master Mix (NEB), resulting in pFS_0399. Oligos FS_1588/FS_1589 were annealed and cloned into pFS_0399 digested with SalI/BamHI, yielding pFS_0400. The Fluc coding sequence was amplified from BbaI712019 (registry of standard biological parts) using FS_1618/FS_1619, digested with BsaI and cloned into BbsI-digested pFS_0400, resulting in pFS_0412, which was used in RNA recording experiments. For each biological replicate, $50 \mathrm{ml}$ of IPTG containing TB medium was inoculated with 22 colonies of E. coli BL21(DE3) transformed with pFS_0271(sfGFP), pFS_0272(Rluc) or pFS_0412(Fluc). When reaching an $\mathrm{OD}_{600}$ of 0.25 , cells were split into $3-\mathrm{ml}$ aliquots in bacterial culture tubes and induced with aTc in case of $\mathrm{P}_{\text {tetA }}$ promoter or $N$-(3-oxododecanoyl)-L-homoserine lactone (3OC6-HSL) (Sigma) in case of $\mathrm{P}_{\text {LuxR }}$ promoter, and cultured in an orbital shaker for 12-14 h at 300 r.p.m., followed by plasmid DNA extraction, SENECA and deep sequencing. Spacers aligning to $s f G F P, R l u c$ and Fluc were quantified as described above (see Data analysis pipeline). The detected number of unique spacers per million sequencing reads was normalized, defining the sum number of spacers per biological replicate as $100 \%$ and plotted using GraphPad Prism v7.0d. For RNArecording with pFS_0271 and pFS_0272 RNA extraction from the same cultures was performed using the RNAsnap method ${ }^{58}$ followed by treatment with the TURBO DNA-free kit (Thermo Scientific) using $1.5 \mu 1$ TURBO DNase to minimize DNA background. Reverse transcription was performed using qScript cDNA SuperMix (Quanta Bio) with 500 ng RNA sample as a template. cDNA was diluted 1:4 and quantification was performed in 2 technical replicates by qRT-PCR using TaqMan Fast Advanced Master Mix (Life Technologies) in a Roche 
LightCycler 96 System. Primers and probes sequences are listed in Supplementary Table 8. Absolute copy number was calculated using standard curve method and 16s rRNA was used as a housekeeper. To determine mRNA copy number corresponding to the number of cells in a single SENECA reaction $\left(6 \times 10^{9}\right)$ was calculated based on the average number of $1870016 s \mathrm{rRNA}$ transcripts per single E. coli cell ${ }^{59}$ (BNID 102992).

\section{Orthogonal synthetic recording}

The Rluc coding sequence was amplified using FS_1620/FS_1137 from pFS_0272 and cloned into pFS_0399 using BbsI-mediated Golden Gate assembly ${ }^{57}$, yielding pFS_0413. The Fluc coding sequence was amplified from Bba_I712019 (registry of standard biological parts) using FS_1621/FS_1619, digested with BbsI and cloned into BsaI-digested pFS_0413, resulting in pFS_0414 which was subsequently used in orthogonal synthetic recording experiments.

For each biological replicate, 33 colonies of $E$. coli BL21(DE3) transformed with pFS_0414, containing (3-Oxododecanoyl)-L-homoserine lactone (3OC6-HSL)-inducible Fluc and aTc-inducible Rluc coding sequences, were inoculated with $50 \mathrm{ml}$ TB medium containing $100 \mu \mathrm{M}$ IPTG. When the reached an $\mathrm{OD}_{600}$ of 0.25 , cells were split into 3-ml aliquots in bacterial culture tubes and induced with $75 \mathrm{ng} / \mathrm{ml}$ of anhydrotetracyclinehydrochloride (aTc) (Cayman Chemical) or $10 \mu \mathrm{M}$ of 3OC6-HSL (Sigma) or a combination of both and cultured in an orbital shaker for $12 \mathrm{~h}$ at 300 r.p.m., followed by plasmid DNA extraction, SENECA, deep sequencing and parallelized RNA extraction from the same culture, followed by reverse transcription and qPCR measurements. Data were analysed as described above for recording of single synthetic transcripts.

\section{Transcriptional response to oxidative stress}

Per biological replicate, 24 colonies of $E$. coli BL21(DE3) transformed with pFS_0235 the evening before (resulting in 1 colony $/ 1.5 \mathrm{ml}$ ) were inoculated with $36 \mathrm{ml}$ IPTG containing TB medium and $100 \mu \mathrm{M}$ IPTG and shaken in a 250 -ml baffled shaker flask until reaching an $\mathrm{OD}_{600}$ of 0.24 to 0.25 . Then cultures were split into $3-\mathrm{ml}$ aliquots into bacterial culture tubes (Grainer) and treated with $\mathrm{H}_{2} \mathrm{O}_{2}(30 \%$ w/w solution, Sigma Aldrich) to a final concentration of $1 \mathrm{mM}$ or an equal volume of $d_{d H_{2}} \mathrm{O}$. Growth was continued for $12 \mathrm{~h}$ at $300 \mathrm{r}$.p.m. followed by harvesting of $2 \mathrm{ml}$ of culture for plasmid DNA extraction, SENECA and deep sequencing. Data were analysed as described above (see Data analysis pipeline). 


\section{Transcriptional response to acid stress}

For $\mathrm{pH}$-controlled growth, potassium-modified lysogenic broth (LB) (10 g/1 tryptone, $5 \mathrm{~g} / 1$ yeast extract, $7.45 \mathrm{~g} / 1 \mathrm{KCl}$ ) was buffered with 100 mM HOMOPIPES (homopiperazine-1,4-bis(2ethanesulfonic acid)). Subsequently, the $\mathrm{pH}$ of the medium was adjusted to either 5.0 (acid stress) or 7.0 (neutral) using $\mathrm{KOH}$ solution as described previously ${ }^{35}$.For each biological replicate, 33 colonies of $E$. coli BL21(DE3) transformed with pFS_0235 (resulting in 1 colony/1.5 mL) were inoculated with $50 \mathrm{ml}$ of pH-adjusted, IPTG-containing LB medium. Samples were harvested at an $\mathrm{OD}_{600}$ of 0.3 to 0.6 for plasmid DNA extraction, SENECA and deep sequencing. Data were analysed as described above (see Data analysis pipeline).

\section{Cloning of aTc-inducible FsRT-Cas1-Cas2 expression construct}

For recording the transcriptional response to paraquat an aTc-inducible FsRT-Cas1-Cas2 expression construct was generated. Therefore, a fragment containing the tet repressor driven by a constitutive promoter and the $\mathrm{P}_{\text {teta }}$ promoter was amplified from pFS_0271 using FS_1574/1575 and digested with BglI/SphI, furthermore the N terminus of FsRT-Cas $1-C a s 2$ was amplified with FS_1576/1577 and digested with SphI/BglII. These two fragments were cloned into BglI/BglII-digested pFS_0235 yielding pFS_0393. The codon-optimized FsRTCas1-Cas2 sequence (Supplementary Data) was obtained from Genscript, amplified using FS_1641/1642 and cloned into pFS_0393 using XhoI/SphI replacing the initial FsRT-Cas1Cas2 coding sequence and yielding pFS_0453.

\section{Transcriptional response to $1 \mathrm{mM}$ or $10 \mathrm{mM}$ paraquat}

Paraquat dichloride hydrate (PESTANAL, Sigma Aldrich) was dissolved at $1 \mathrm{M}$ in $\mathrm{ddH}_{2} \mathrm{O}$. For each biological replicate, 50 colonies of E. coli BL21(DE3) transformed with pFS_0393 were inoculated with $75 \mathrm{ml} \mathrm{TB}$ medium containing $30 \mathrm{ng} / \mathrm{ml}$ aTc and shaken in baffled shaker flasks until they reached an $\mathrm{OD}_{600}$ of 0.24 to 0.25 . Then cultures were split into 3-ml aliquots into bacterial culture tubes and treated with either $1 \mathrm{mM}$ or $10 \mathrm{mM}$ paraquat and cultured for an additional 11-12 $\mathrm{h}$ before harvesting of $2 \mathrm{ml}$ of culture for plasmid DNA extraction, SENECA and deep sequencing. Data were analysed as described above (see Data analysis pipeline).

\section{Transcriptional response to transient paraquat exposure}

For each biological replicate two colonies of E. coli BL21(DE3) transformed with pFS_0453 were inoculated with $3 \mathrm{ml}$ of TB medium containing $30 \mathrm{ng} / \mathrm{ml}$ aTC in standard bacterial culture 
tubes. For the first $12 \mathrm{~h}$ all cultures were cultivated in the absence of paraquat (300 r.p.m., 37 $\left.{ }^{\circ} \mathrm{C}\right)$. Then $2 \mathrm{ml}$ of culture was aspirated, while the remaining $1 \mathrm{ml}$ was spun down $(2,300 \mathrm{~g}, 10$ min) the supernatant was aspirated and the bacterial pellet resuspended in $3 \mathrm{ml}$ of fresh TB medium containing $30 \mathrm{ng} / \mathrm{ml}$ of aTc. For both transient and permanent stimulus conditions, paraquat was added to $10 \mathrm{mM}$ final concentration and the cultures were grown for an additional $12 \mathrm{~h}$ as above. Then $2 \mathrm{ml}$ of culture was removed, the remaining $1 \mathrm{ml}$ was pelleted as above and resuspended in $3 \mathrm{ml}$ of fresh TB medium containing $30 \mathrm{ng} / \mathrm{ml}$ of aTc. Paraquat was added to 10 $\mathrm{mM}$ for the permanent stimulus condition and cultures were grown for an additional $12 \mathrm{~h}$ as above. Then $2 \mathrm{ml}$ of culture was harvested for plasmid DNA extraction, SENECA and deep sequencing. Additionally, $100 \mu \mathrm{l}$ of culture was harvested for RNA extraction by the RNASnap protocol as described above followed by treatment with the TURBO DNA-free Kit (Thermo Scientific) using $1.5 \mu$ of TURBO DNase. Ribosomal RNA was depleted using Ribo-Zero rRNA Removal Kit (Illumina) followed by library prep using TruSeq Stranded mRNA (Illumina) and deep sequencing on an NextSeq 500/550 High Output v2 kit (75 cycles) sequencing each library at a depth of 4 million reads or greater.

\section{Code availability}

The custom scripts used for the described data analysis are available on the Platt Laboratory website (www.platt.ethz.ch).

\section{Data availability}

Deep sequencing data are available in the National Center for Biotechnology Information Sequence Read Archive (PRJNA484149). The data sets generated and/or analysed during the current study are available from the corresponding author upon reasonable request.

46. Finn, R. D., Clements, J. \& Eddy, S. R. HMMER web server: interactive sequence similarity searching. Nucleic Acids Res. 39, W29-W37 (2011).

47. Biswas, A., Staals, R. H. J., Morales, S. E., Fineran, P. C. \& Brown, C. M. CRISPRDetect: A flexible algorithm to define CRISPR arrays. BMC Genomics 17, 356 (2016). 
48. Zhang, Q. \& Ye, Y. Not all predicted CRISPR-Cas systems are equal: isolated cas genes and classes of CRISPR like elements. BMC Bioinformatics 18, 92 (2017).

49. Edgar, R. C. MUSCLE: multiple sequence alignment with high accuracy and high throughput. Nucleic Acids Res. 32, 1792-1797 (2004).

50. Bolger, A. M., Lohse, M. \& Usadel, B. Trimmomatic: a flexible trimmer for Illumina sequence data. Bioinformatics 30, 2114-2120 (2014).

51. Langmead, B. \& Salzberg, S. L. Fast gapped-read alignment with Bowtie 2. Nat. Methods 9, 357-359 (2012).

52. Li, H. et al. The Sequence Alignment/Map format and SAMtools. Bioinformatics 25, 2078-2079 (2009).

53. Wang, L., Wang, S. \& Li, W. RSeQC: quality control of RNA-seq experiments. Bioinformatics 28, 2184-2185 (2012).

54. Crooks, G. E., Hon, G., Chandonia, J. M. \& Brenner, S. E. WebLogo: a sequence logo generator. Genome Res. 14, 1188-1190 (2004).

55. Quinlan, A. R. \& Hall, I. M. BEDTools: a flexible suite of utilities for comparing genomic features. Bioinformatics 26, 841-842 (2010).

56. Liao, Y., Smyth, G. K. \& Shi, W. featureCounts: an efficient general purpose program for assigning sequence reads to genomic features. Bioinformatics 30, 923-930 (2014).

57. Engler, C., Gruetzner, R., Kandzia, R. \& Marillonnet, S. Golden gate shuffling: a onepot DNA shuffling method based on type IIs restriction enzymes. PLoS One 4, e5553 (2009).

58. Stead, M. B. et al. RNAsnap ${ }^{\mathrm{TM}}$ : a rapid, quantitative and inexpensive, method for isolating total RNA from bacteria. Nucleic Acids Res. 40, e156 (2012).

59. Milo, R., Jorgensen, P., Moran, U., Weber, G. \& Springer, M. BioNumbers--the database of key numbers in molecular and cell biology. Nucleic Acids Res. 38, D750D753 (2010). 
a

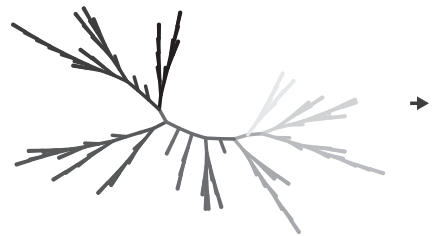

identify RT-Cas1 orthologs and select divergent subset

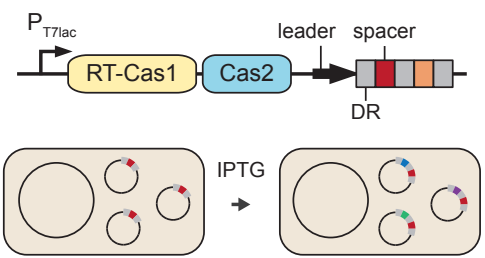

heterologuosly express codon optimized RT-Cas1 and Cas2 with corresponding CRISPR array in E. coll

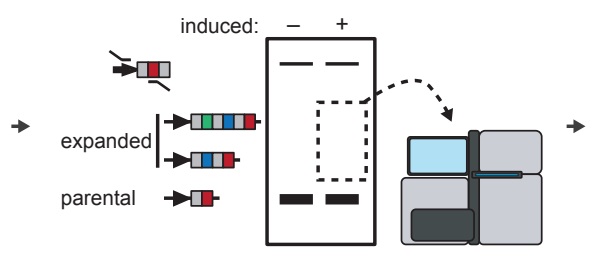

amplify CRISPR array, size select, and sequence expanded arrays reads

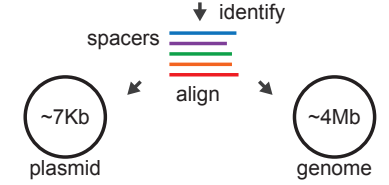

identify novel spacers and align to plasmid and genome

b

Unknown Domain Cas6 homology

Consensus

Host

$\underline{0.2}$

Candidatus Accumulibacter sp. BA-91 Fusicatenibacter saccharivorans Bacteroides fragilis strain S14 Campylobacter fetus subsp. Fetus Marinomonas mediterrane

Teredinibacter turnerae T8412 Woodsholea maritima

Desulfarculus baarsii DSM 2075 Azospirillum lipoferum $4 B$

Cellulomonas bogoriensis 69B4

Micromonospora rosaria

Candidatus Accumulibacter sp. SK-02

Tolypothrix campylonemoides

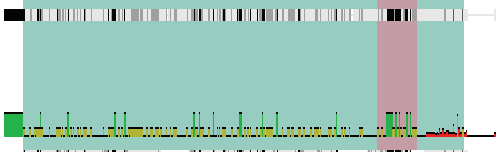

-

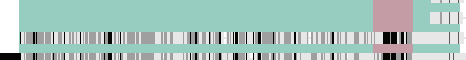

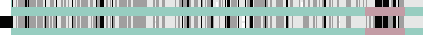

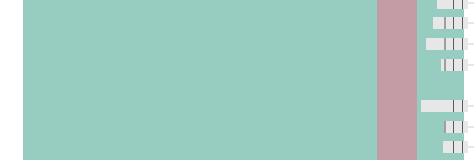

RT

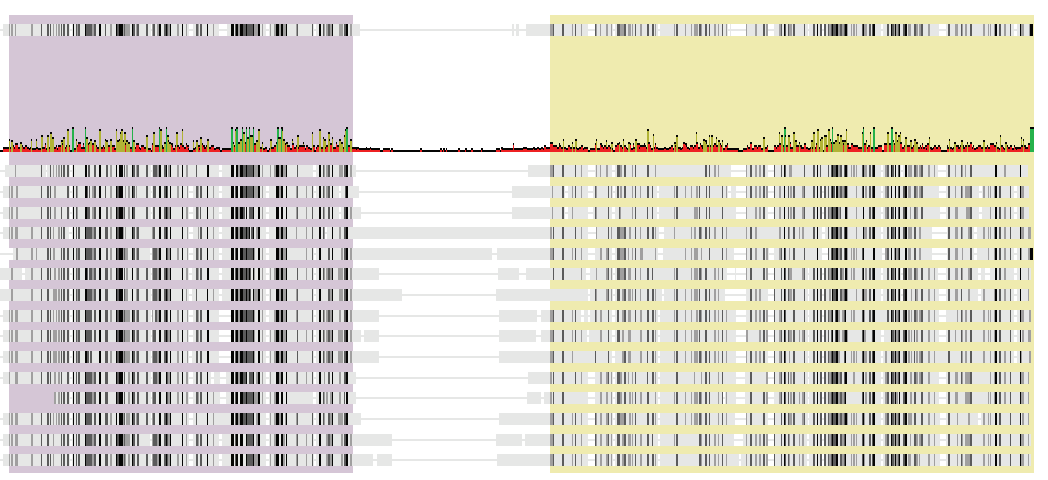

C

Candidatus Accumulibacter sp. BA-91

Fusicatenibacter saccharivorans Fusicatenibacter saccharivorans ARRAY2

Eubacterium saburreum DSM 3986 Eubacterium saburreum DSM 3986 ARRAY2 Bacteroides fragilis strain S14

Campylobacter fetus subsp. Fetus Teredinibacter turnerae T8412 Woodsholea maritima Desulfarculus baarsii DSM 2075 Azospirillum lipoferum $4 B$ Azospirillum lipoferum 4B ARRAY2 Cellulomonas bogoriensis 69B4 Micromonospora rosaria

Micromonospora rosaria ARRAY2 Candidatus Accumulibacter sp. SK-02 Tolypothrix campylonemoides Oscillatoriales cyanobacterium

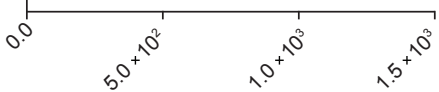

aligning spacers per million reads (log-scale) d

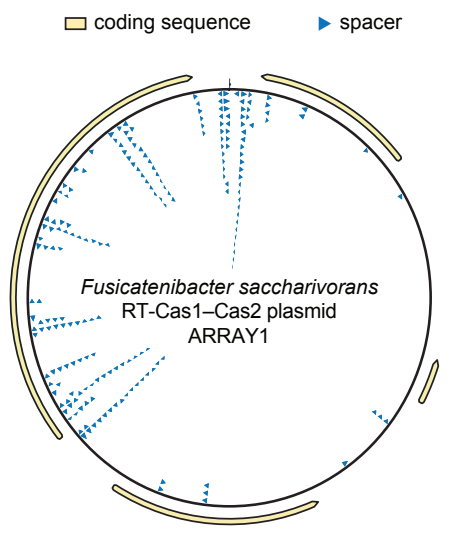

e

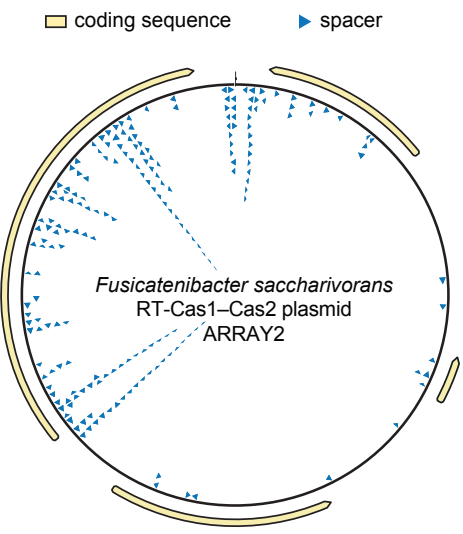


Extended Data Figure 1 | RT-Cas1 orthologue search and screening. a, Experimental workflow involving the identification of $121 \mathrm{RT}-\mathrm{Cas} 1$ orthologues, overexpression in E. coli from the plasmid carrying minimal CRISPR array, containing leader-DR-spacer1-DR-spacer2DR, followed by deep sequencing of expanded CRISPR arrays, and analysis and characterization of identified spacers. b, A comparison of the 14 disparate RT-Cas1 proteins selected for functional testing. Indicated on the left is the host species followed by a neighbour-joining phylogenetic tree built using Jukes-Cantor genetic distances of a MUSCLE multiple sequence alignment. The large 'unknown domain' is highlighted in green, Cas6 homology domain in pink, RT domain in purple, and Cas1 in yellow. c, Detection frequency of newly acquired spacers after overnight growth and induction of RT-Cas1-Cas2 in E. coli BL21(DE3) in different induction media. Shown is the sum of spacer counts per 1 million sequencing reads, $n=1$ biological sample. d, Representative alignments of 200 spacers sequenced from $F$. saccharivorans array 1 to the corresponding overexpression plasmid. e, Representative alignments of 200 spacers sequenced from $F$. saccharivorans array 2 to the corresponding overexpression plasmid. 


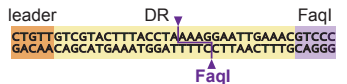
Fâql

$$
\downarrow \text { Faql digest }
$$

I leader DR forward primer CTGTGGTGGACTIAACCTA
GACAACAGCATGAAATGGATITTC

+ annealed adapters

Illumina P7 3' adapter TGAGAGATCGEAAGAGCACACGTCTEAACTCCAGTCAC
ICTAGCCTICTCGTGGGAGATITAGGTAGTE
reverse primer

$$
\downarrow \begin{gathered}
\text { adapter ligation by } \\
\text { Golden Gate Assembly }
\end{gathered}
$$

IVa

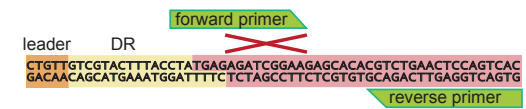

$\mathrm{Va}$

$$
\downarrow \text { PCR-amplification }
$$

b

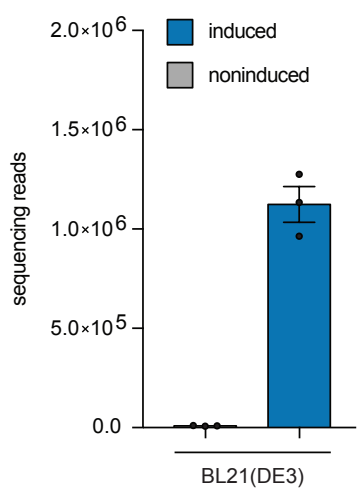

no exponential PCR product c

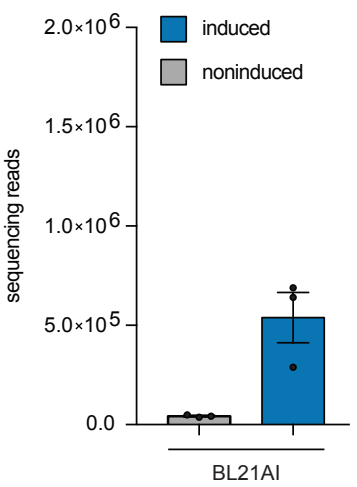

spacer acquisition

II

III

IVb leader DR spacer

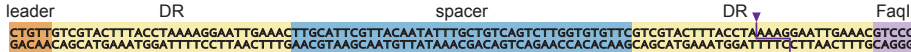

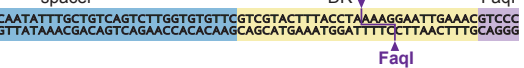

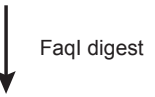

leader DR forward primer

spacer DR forward primer

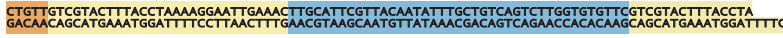

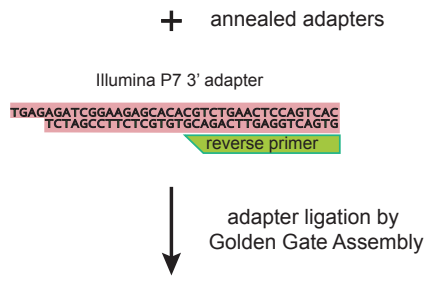

\section{leader DR forward primer $\quad$ spacer}

forward primer

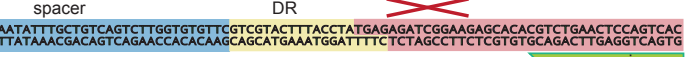
PCR-amplification

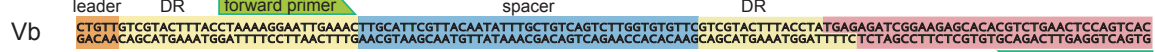

exponential PCR amplification of newly acquired spacer

d

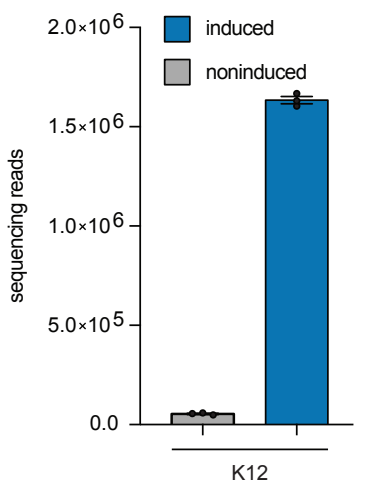

e

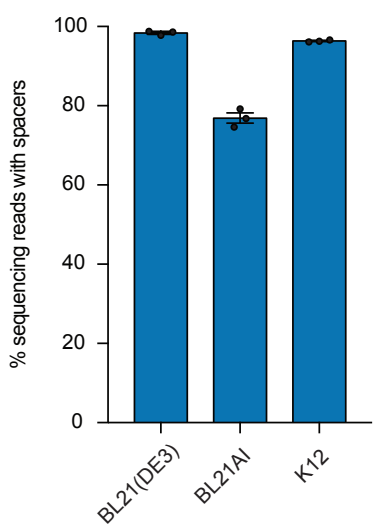

f

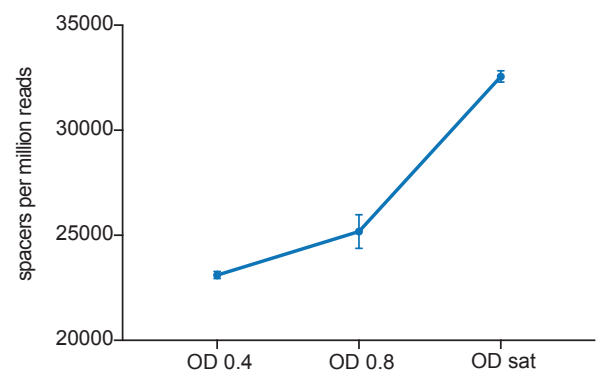

g

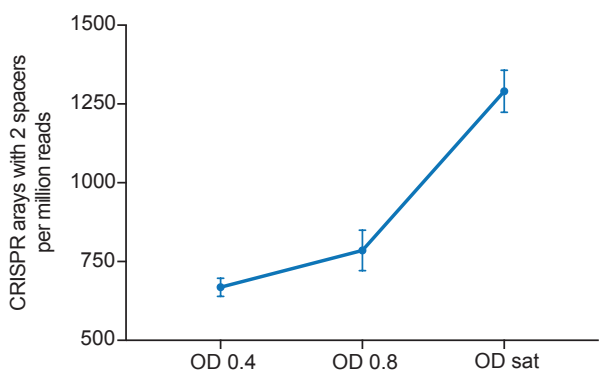


Extended Data Figure 2 | SENECA workflow and assessment of Record-seq efficiency in different culture conditions. a, SENECA relies on a plasmid containing a minimal CRISPR array consisting of the leader sequence followed by a single direct repeat and a recognition sequence for the restriction enzyme FaqI. The SENECA workflows for the (left) parental and (right) expanded arrays are shown. In a Golden Gate reaction, FaqI cleaves within the direct repeat (I/II), introducing sticky ends for ligation to an Illumina P7 3' adaptor (III). For the parental array this results in a single truncated direct repeat (IVa). For the expanded array this results in a truncated direct repeat as well as an intact direct repeat and spacer (IVb). PCR with primers binding to the full-length direct repeat and the Illumina P7 3' adaptor results in linear amplification of the parental array (Va) and exponential amplification of the expanded array (Vb). b, Sequencing reads obtained from E. coli BL21(DE3) cells transformed with FsRT-Cas1Cas2-encoding plasmid with or without IPTG induction. c, As in b but in E. coli BL21AI. d, As in b but in $E$. coli NovaBlue(DE3), a K12 substrain of E. coli. e, Percentage of sequencing reads from induced samples containing newly acquired spacers. f, Spacers per million sequencing reads obtained from cultures at an $\mathrm{OD}_{600}$ of $0.4,0.8$ or upon saturation. $\mathbf{g}$, CRISPR arrays with two spacers per million sequencing reads obtained from cultures at an $\mathrm{OD}_{600}$ of $0.4,0.8$ or upon saturation Values in $\mathbf{b}-\mathbf{g}$ are mean \pm s.e.m., $n=3$ independent biological samples. 


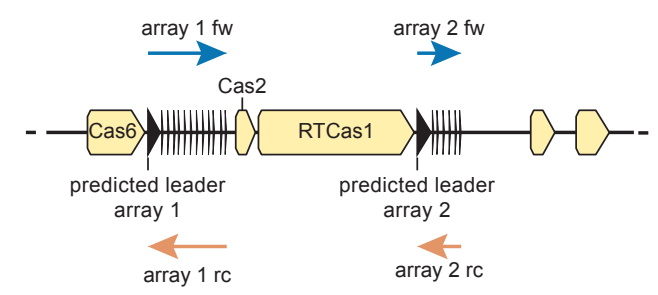

Fusicatenibacter saccharivorans

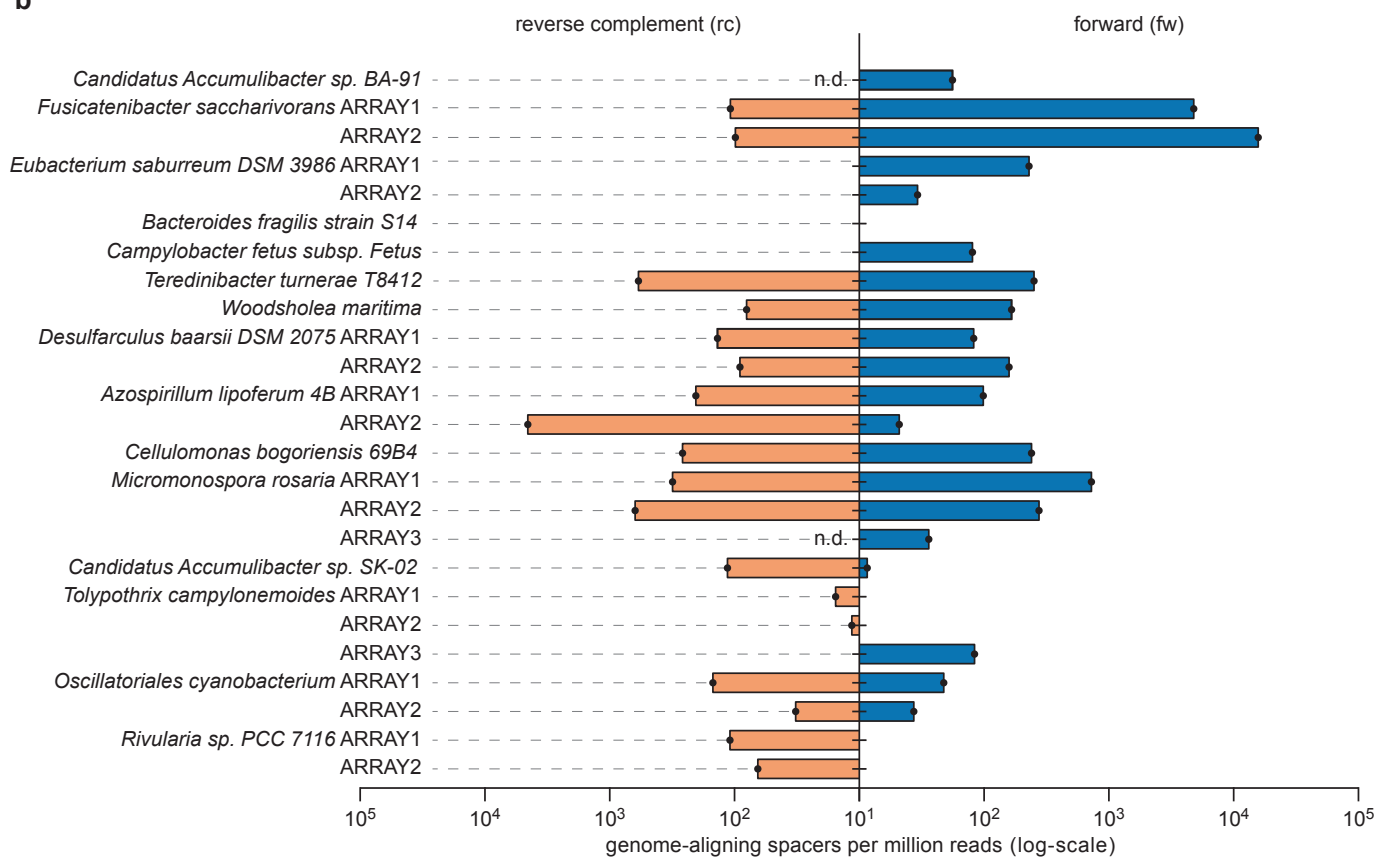




\section{Extended Data Figure 3 | Record-seq-based screen of RT-Cas1 orthologues and CRISPR}

array directionalities. a, Schematic of the $F$. saccharivorans CRISPR locus depicting the selection of CRISPR arrays and directionalities for Record-seq analysis. CRISPR arrays within each locus were identified and cloned into plasmids encoding corresponding RT-Cas1-Cas2 coding sequences. Arrays were tested in both possible directionalities, forward and reverse with a 150-bp leader. In cases of insufficient genomic data, arrays were tested in only one directionality. b, Record-seq readout of RT-Cas1 orthologues and CRISPR array directionalities. Acquisition efficiencies for forward (fw) and reverse complement (rc) directionality of each array are plotted in blue and orange, respectively. Values are genomealigning spacers per million sequencing reads, $n=1$ biological sample. 

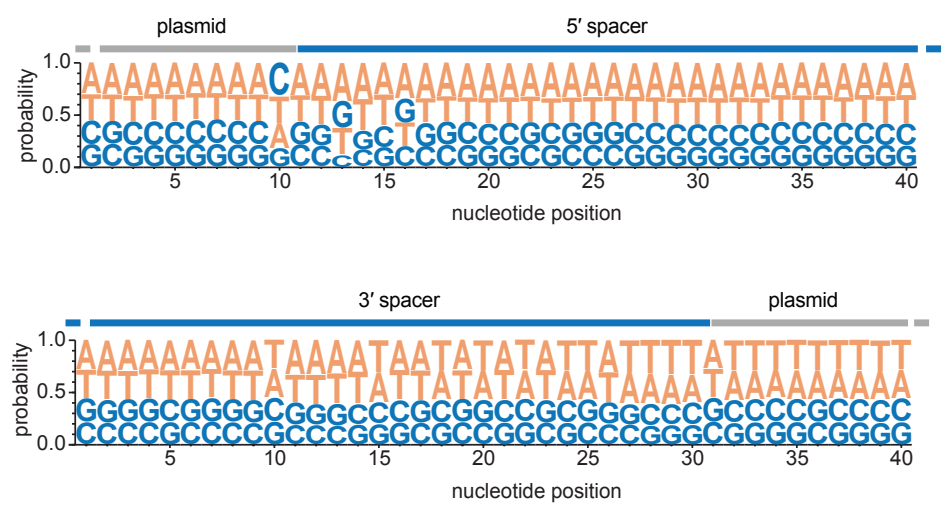

c

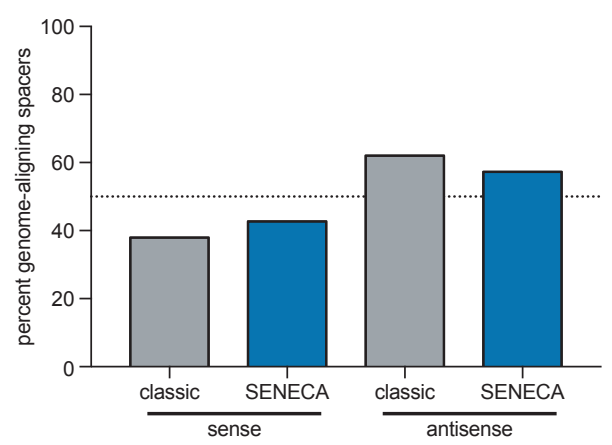

f

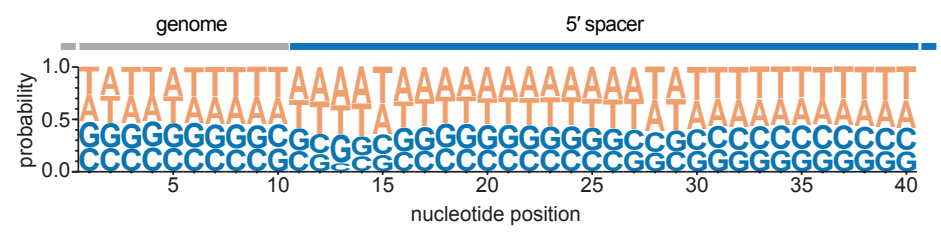

3' spacer

genome

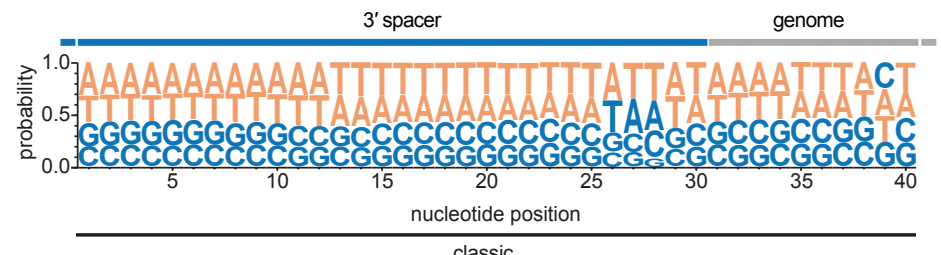

b

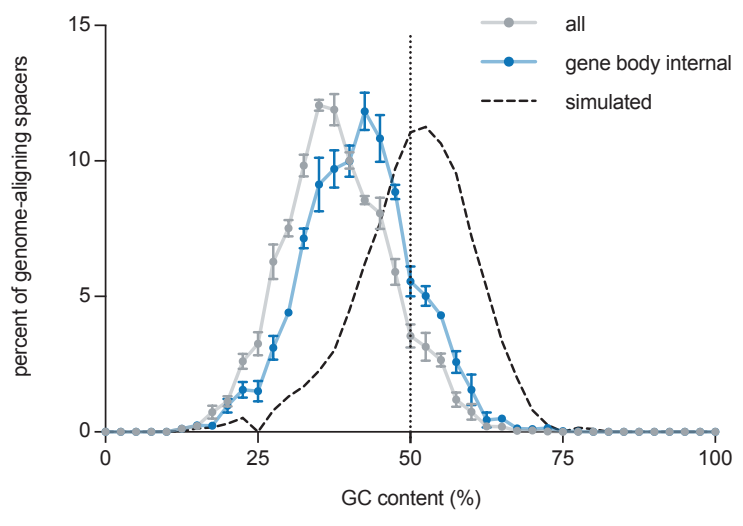

e

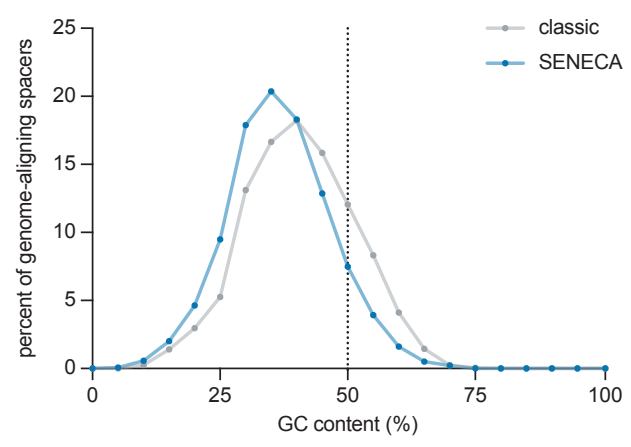

h

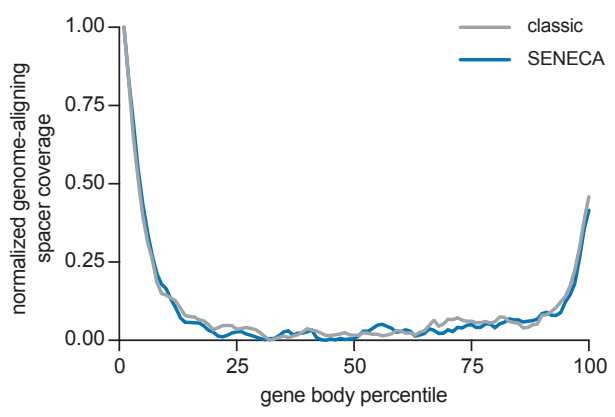




\section{Extended Data Figure 4 | Characterization of spacers acquired by FsRT-Cas1-Cas2 and}

comparison of SENECA and classic spacer acquisition readouts. a, Nucleotide probabilities determined using plasmid-aligning spacers merged across $n=14$ independent biological samples, prepared as for Fig. 2f. b, Histogram of spacer GC content for all spacers or spacers acquired internal to the body of the transcript ('gene body internal'). Values represent mean percentage of genome-aligning spacers \pm s.e.m., $n=3$ independent biological samples. c, Percentage of spacers aligning to either the sense or antisense strand of coding genes. The sense or antisense orientation label is with respect to the RNA, prepared as for Fig. 2c. d, Length distribution of genome-aligning spacers, prepared analogous to Fig. 2d. e, GC content distribution of genome-aligning spacers. The dotted line represents a balanced (50\%) GC content, prepared as for Fig. 2e. f, Nucleotide probabilities for classic acquisition readout, prepared as for Fig. 2f. g, Nucleotide probabilities for SENECA acquisition readout, prepared analogous to Fig. $2 \mathrm{f}$. Gene body coverage. For each gene the spacer coverage was determined and transformed into percentiles for comparison. Values are mean normalized coverage. $n=1$ pooled sample, containing 5,798 spacers. Values in $\mathbf{c}-\mathbf{g}$ are mean percentage of genome-aligning spacers, $n=1$ pooled sample, containing 5,798 spacers. 


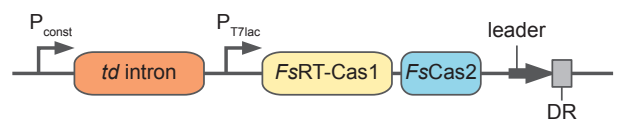

$t d$ intron splice junction recording
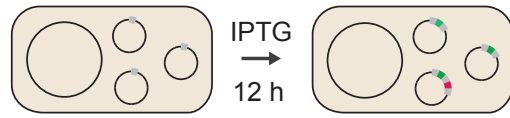

E. coli BL21(DE3)

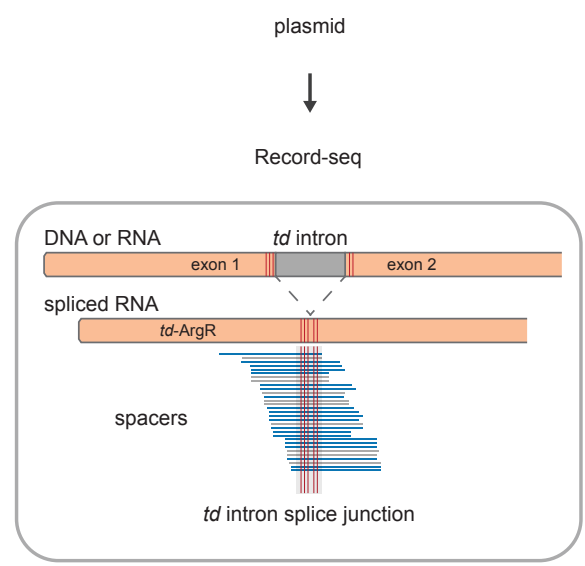

d

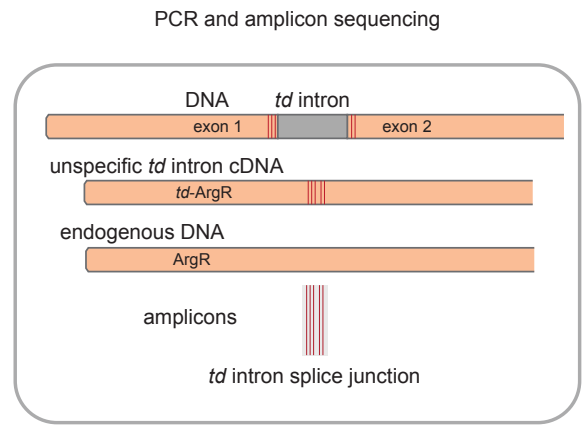

e

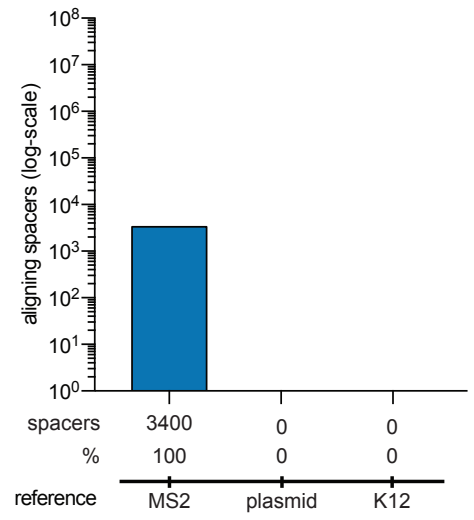

b

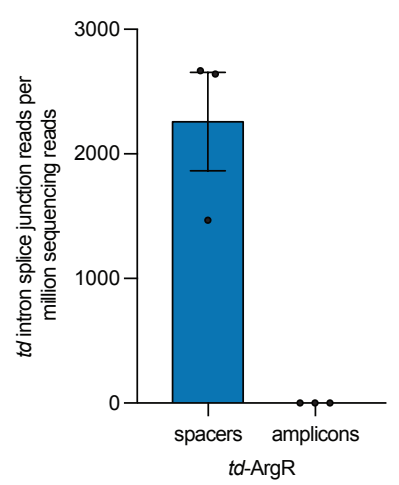

C

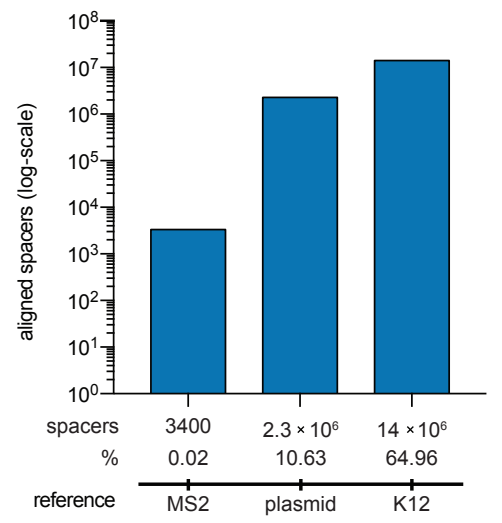

f
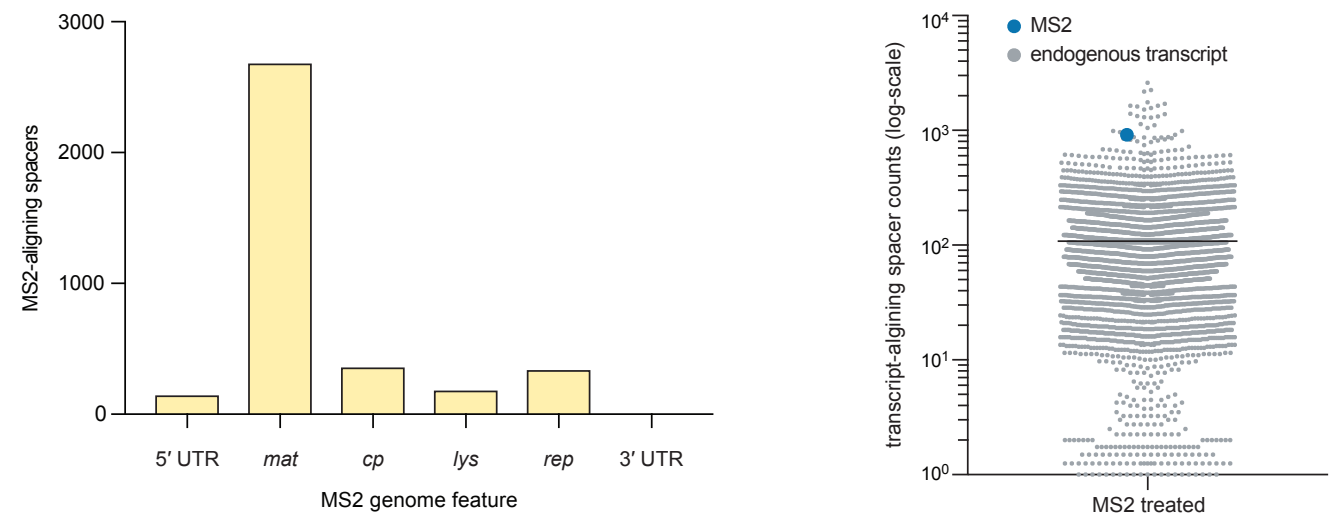


\section{Extended Data Figure 5 | Characterization of spacers acquired by FsRT-Cas1-Cas2. a,}

Experimental workflow for determining the specificity of FsRT-Cas1-Cas2 for RNA using the $t d$ intron splice junction to detect RNA-derived spacers. Genomic DNA (gDNA) was extracted from an independent culture and subjected to targeted deep sequencing of the $t d$ intron insertion site. b, Quantification of $t d$ intron splice junctions. The splice junction is specific to RNAderived spacers and not genomic DNA or cDNA copies generated by alternative reverse transcriptases in the $E$. coli genome. Values represent mean $t d$ intron splice junction counts per million sequencing reads \pm s.e.m., $n=3$ independent biological samples. c, Number of spacers aligned to plasmid, E. coli genome, and MS2 genome, showing CRISPR acquisition from an RNA virus. The total number and per cent of spacers aligning to each reference are shown. Values represent the sum of MS2-aligning spacers across replicates, $n=64$ technical replicates from $n=2$ biological samples, representing 22 million spacers. d, Number of MS2-aligned spacers from $\mathbf{c}$ that align to the overexpression plasmid, E. coli and MS2 genome, showing that MS2-aligned spacers are specific to the MS2 genome. The total number and percentage of MS2aligned spacers that subsequently align to each reference are shown, $n=64$ technical replicates from $n=2$ biological samples, representing 22 million spacers. e, Total number of spacers aligning to features of the MS2 genome, $n=64$ technical replicates from $n=2$ biological samples, representing 22 million spacers. f, Scatter plot of transcript counts from the MS2 and $E$. coli genomes. Each dot represents the mean spacer count for each transcript, $n=4$ independent biological samples. The horizontal black bars are mean genome-aligning spacer count across all transcripts \pm s.e.m. 
aTc [ng/mL]

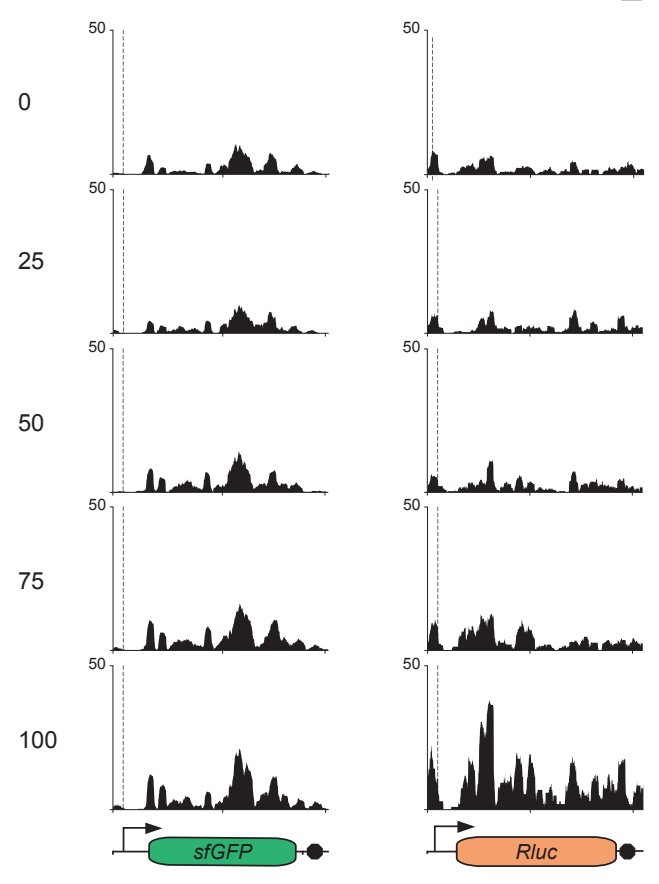

f

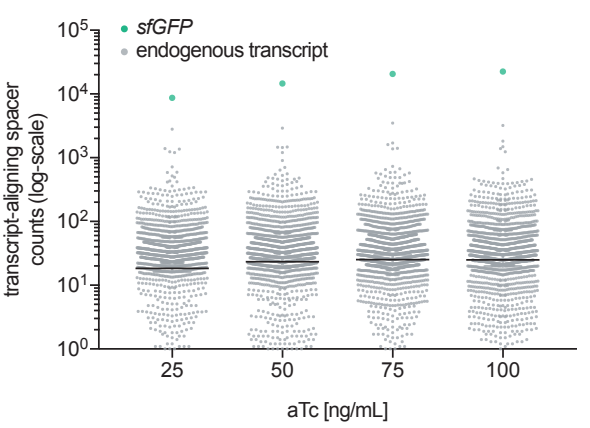

h

Fluc

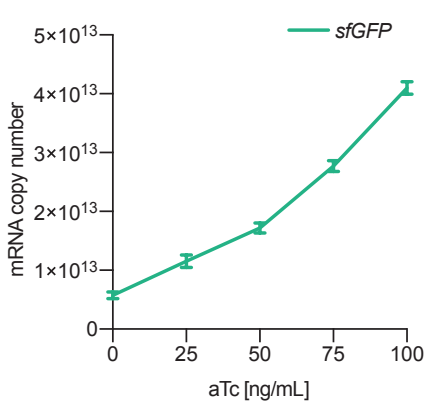

d

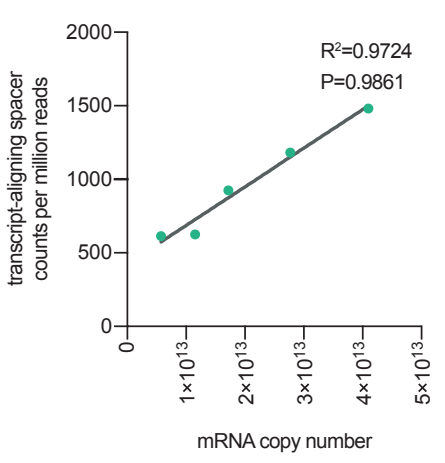

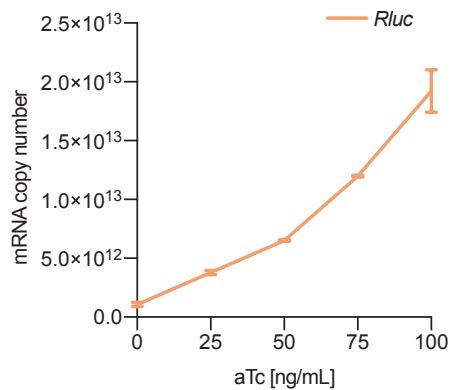

e

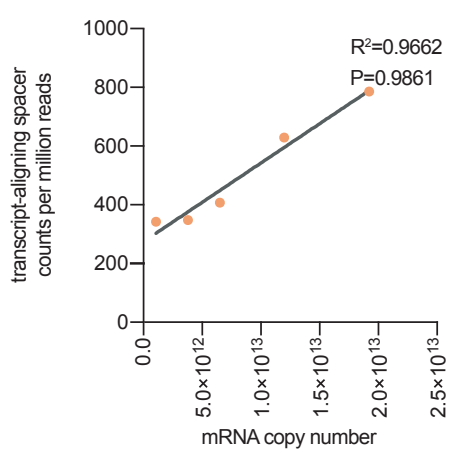

g

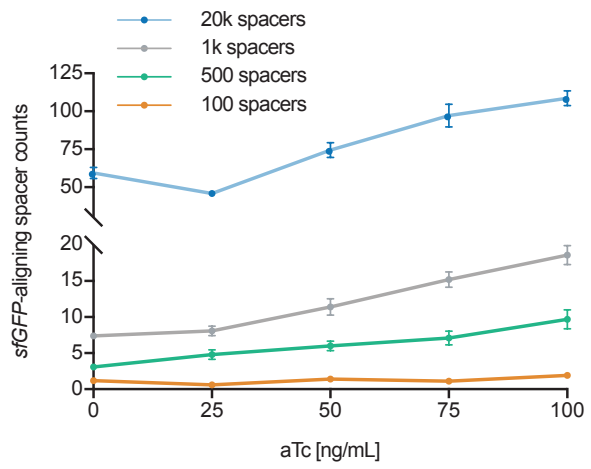

i

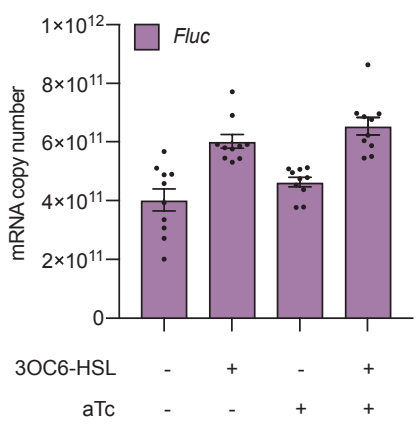

j

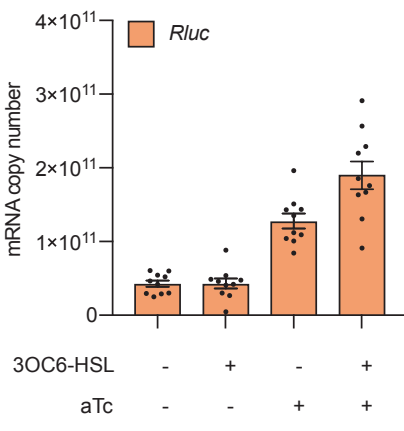




\section{Extended Data Figure 6 | Quantitative analysis of arbitrary RNA sequence recording using}

qRT-PCR and Record-seq. a, Coverage of spacers from Fig. $3 \mathrm{f}$ aligning to $s f G F P$ or Rluc. Arrow and dotted line reflect the transcription start site (TSS), black octagon indicates the transcriptional terminator. For each nucleotide position, the sum spacer coverage per million sequencing reads is shown, $n=10$ independent biological samples. $\mathbf{b}$, Absolute quantification of $s f G F P$ mRNA measured by qRT-PCR. Samples from Fig. 3f. Values are mean \pm s.e.m. copy number per $6 \times 10^{9}$ cells, normalized by $16 S$ rRNA copy number, $n=10$ independent biological samples. c, As in b, but for Rluc. d, Scatter plot depicting the correlation between absolute $s f G F P$ mRNA copy number and the number of transcript-aligning spacers from Fig. 3f. Linear regression fit, coefficient of determination $\left(R^{2}\right)$, and Pearson linear correlation coefficient $(P)$, $n=10$ independent biological samples. e, As in $\mathbf{d}$, but for Rluc. f, Comparison of spacer counts for arbitrary $s f G F P$ sequence and endogenous transcripts. Each dot represents the mean spacer count for each transcript, horizontal black bars are mean genome-aligning spacer count \pm s.e.m., $n=10$ independent biological samples. g, Dose-response relationship between $s f G F P$-aligning spacers and inducer concentration for different numbers of recorded spacers. These data represent the average number of $s f G F P$-aligning spacers \pm s.e.m., $n=10$ independent biological samples. h, Relative spacer count of spacers mapping to the Fluc transcript after 3OC6-HSL induction. Values are the normalized mean number of spacers per million sequencing reads \pm s.e.m. with $n=6$ independent biological samples. i, Absolute quantification of Fluc mRNA measured by qRT-PCR. Data were obtained from the same bacterial cultures as in Fig. $3 \mathrm{~g}$. Values are mean copy number per $6 \times 10^{9}$ cells, normalized by $16 S \mathrm{rRNA}$ copy number, \pm s.e.m., $n=10$ independent biological samples. $\mathbf{j}$, As in $\mathbf{g}$, but for Rluc. 
a
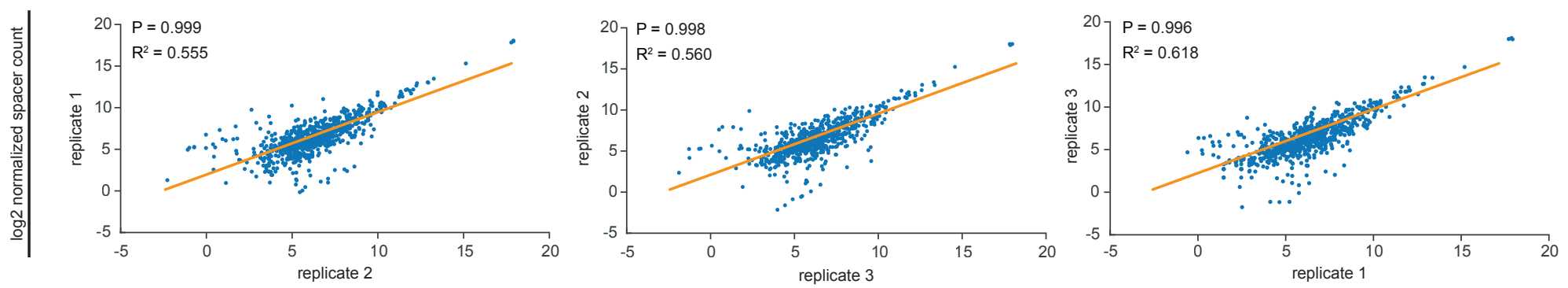

b
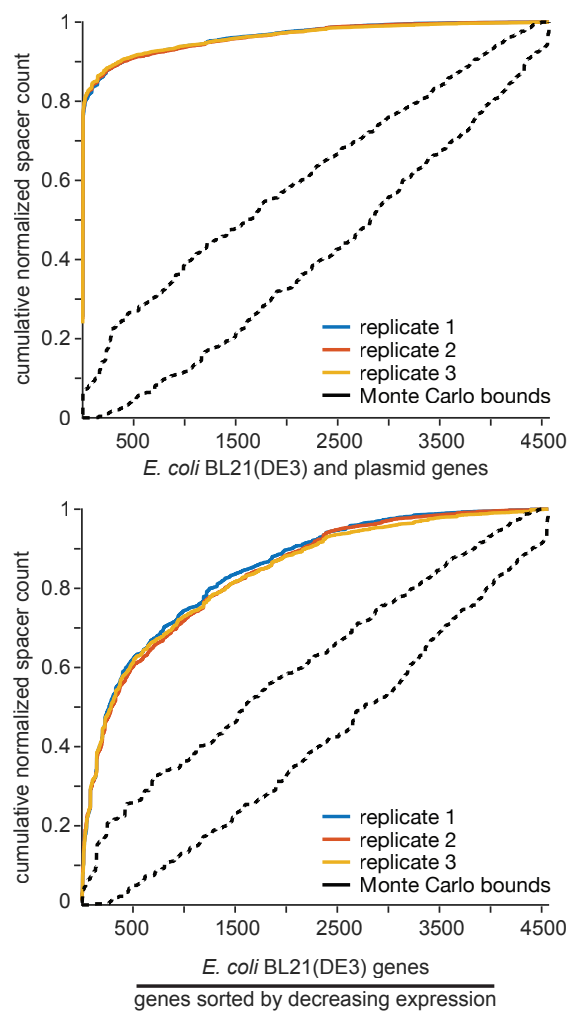

log2 normalized spacer count

c
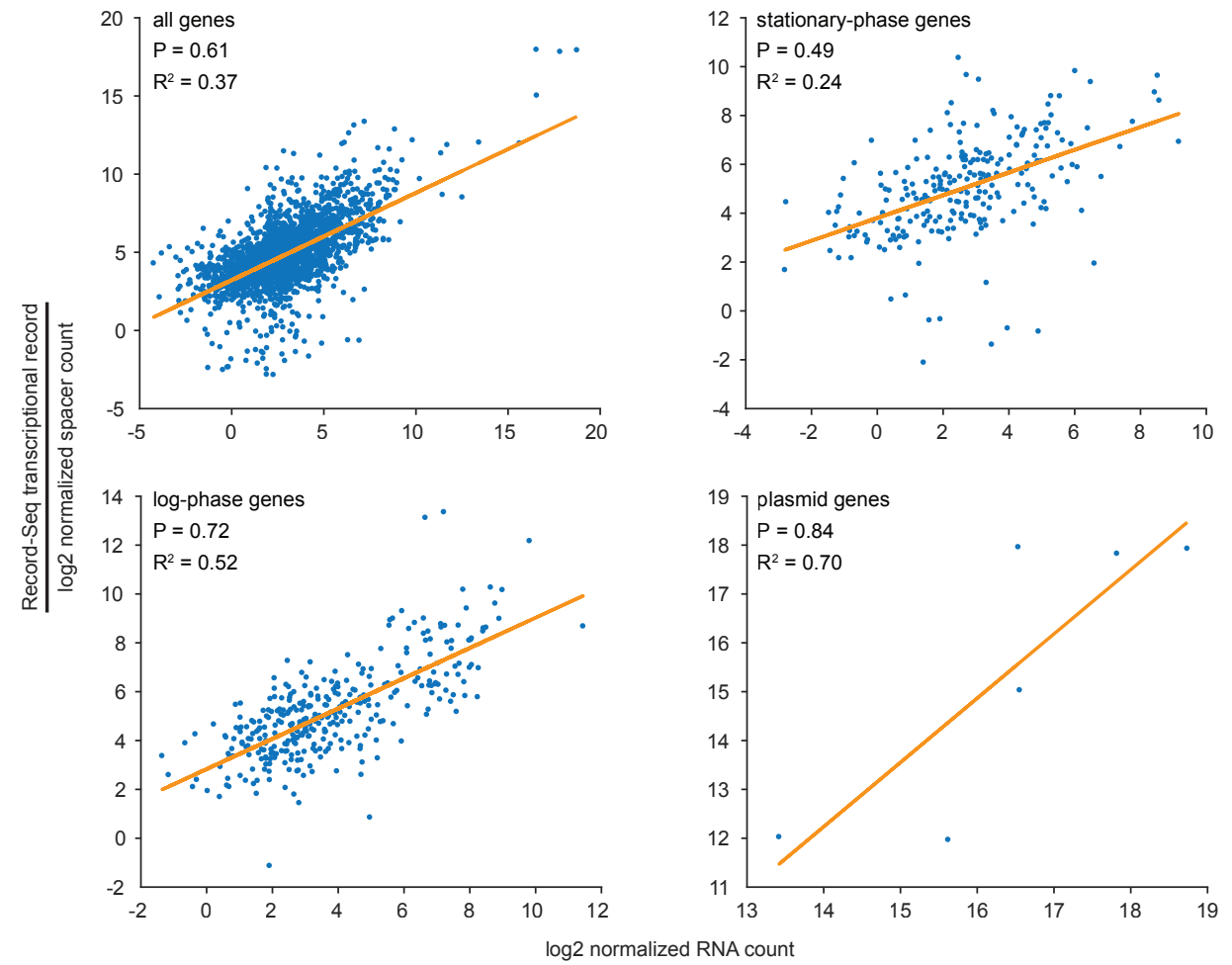

$\overline{\text { RNA-seq stationary phase snapshot }}$

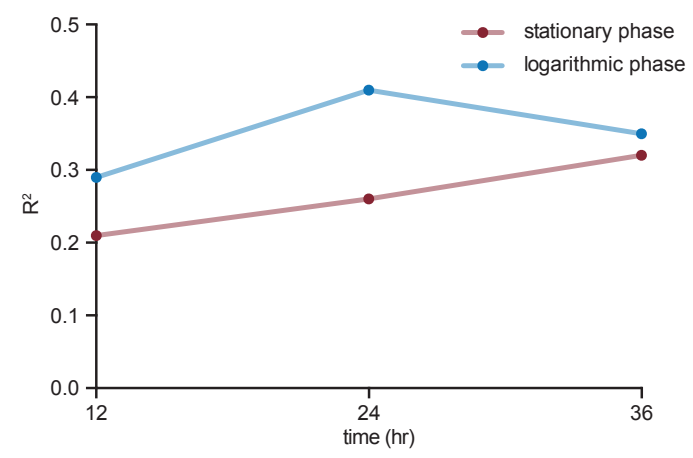

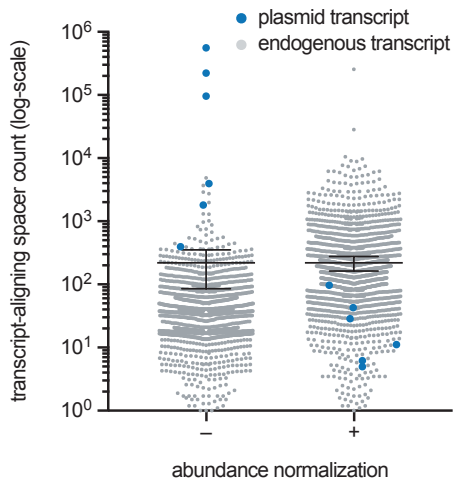




\section{Extended Data Figure 7 | Record-seq reveals cumulatively highly expressed genes. a,}

Scatter plots depicting Record-seq correlation between $n=3$ independent biological replicates shown in $\mathbf{b}$ and $\mathbf{c}$. Linear regression fit, coefficient of determination $\left(R^{2}\right)$, and Pearson linear correlation coefficient $(P)$ are shown for each comparison. Data represent $\log _{2}$-normalized transcript quantification counts. b, Spacers are preferentially acquired from highly expressed genes. Record-seq spacer counts for plasmid and E. coli genes (top) or only E. coli genes (bottom) according to decreasing RNA-seq-based gene expression values. Monte Carlo bounds reflect simulated spacers with no transcriptional bias. Mean cumulative normalized spacer count, and Monte Carlo bounds are shown, $n=3$ independent biological samples. c, Assessing the correlation between an RNA-seq stationary phase snapshot and a Record-seq transcriptional record. RNA-seq and Record-seq were performed on the same population of E. coli BL21(DE3) in stationary phase growth, induced to express FsRT-Cas1-Cas2 overnight. The correlation between all (top left), stationary-phase (top right), log-phase (bottom left), and plasmid-borne (bottom right) genes are shown. The linear regression fit, coefficient of determination $\left(R^{2}\right)$, and Pearson linear correlation coefficient $(P)$ are shown for each comparison. The data represent the $\log _{2}$ normalized transcript quantification counts averaged across replicates, $n=3$ independent biological samples. d, Correlation of Record-seq with log- and stationary-phase genes over longterm cultivation. These data represent the $R^{2}$ value calculated as described for $\mathbf{b}$ for either stationary or logarithmic phase gene sets using different $E$. coli culture time points as inputs with $n=3$ independent biological samples. e, Comparison of transcript-aligning spacer counts with and without normalizing for gene expression level. Each dot represents the mean normalized number of counts per transcript with $n=3$ independent biological samples. The horizontal black bars are mean genome-aligning spacer count \pm s.e.m. See Supplementary Notes for detailed discussions on $\mathbf{b}, \mathbf{d}$. 

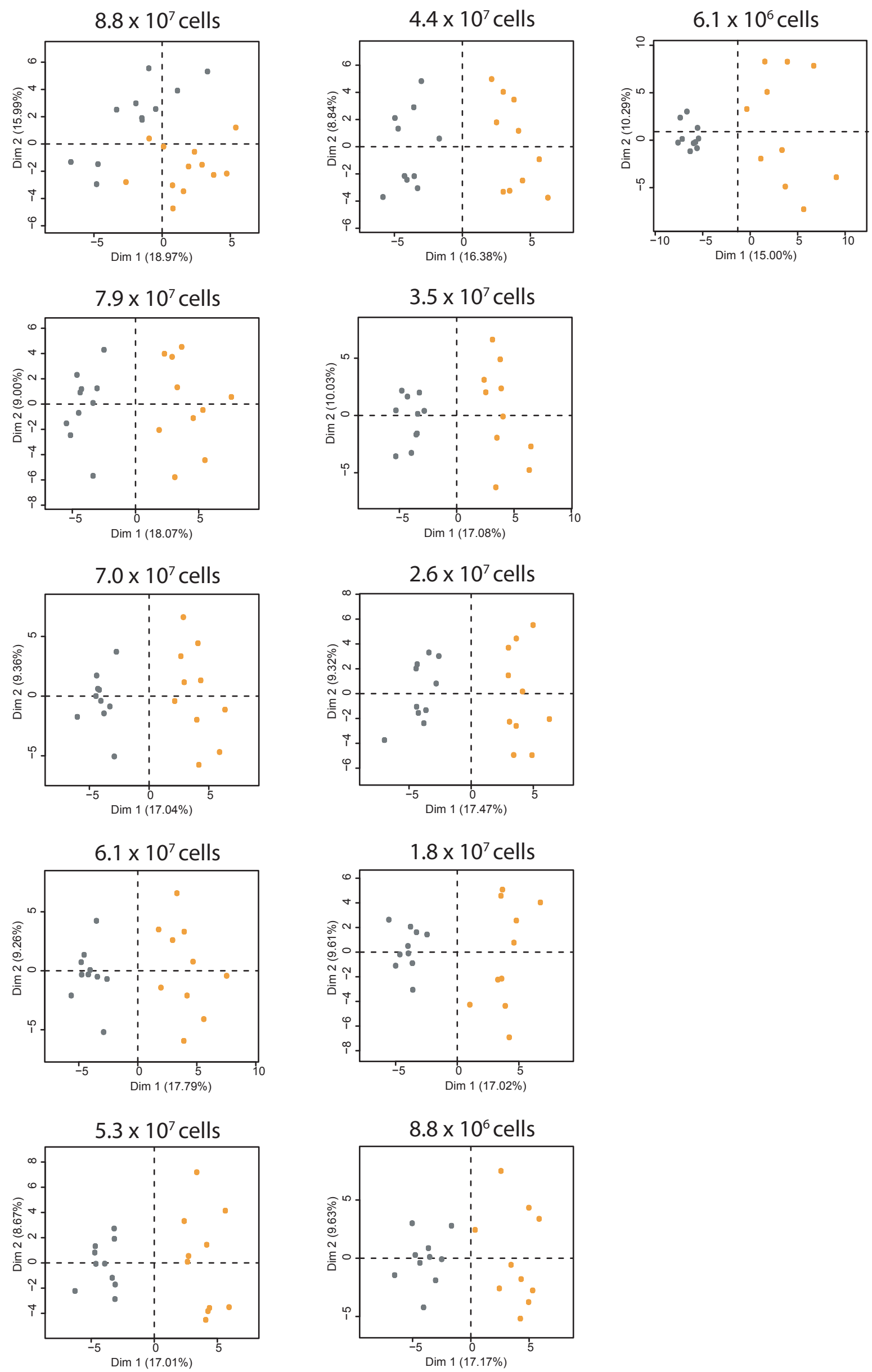


\section{Extended Data Figure 8 | Defining the minimum number of cells required for assessing}

complex cellular behaviours using Record-seq and PCA. Using the acid stress response data set shown in Fig. 4, PCA was performed on the entire data set as well as progressively and randomly downsampled data. These data show that Record-seq appropriately classifies the acid stress response samples with $7 \%$ of the original data (corresponding to 314 spacer or $6.1 \times 10^{6} \mathrm{E}$. coli cells). The calculation of the required number of $E$. coli cells is described in detail in Supplementary Notes; $n=10$ independent biological samples. 


\section{$8.8 \times 10^{7}$ cells}

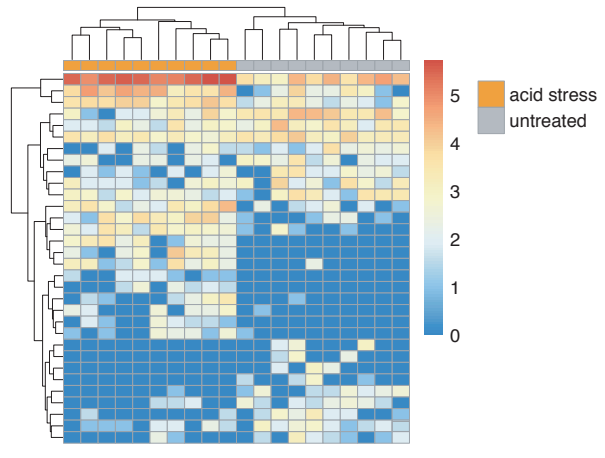

$7.9 \times 10^{7}$ cells

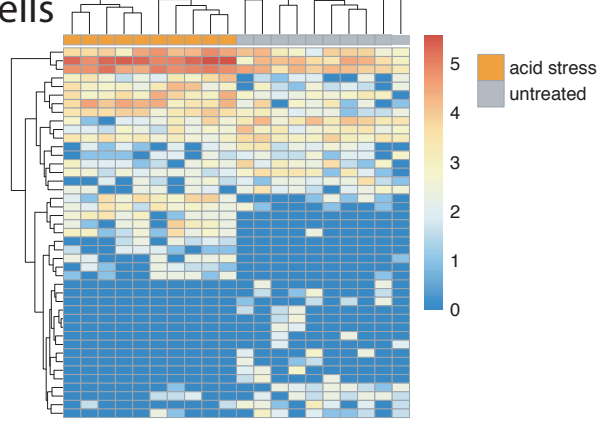

$7.0 \times 10^{7}$ cells

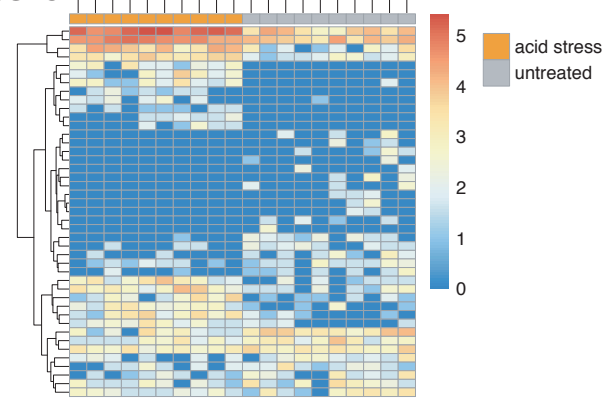

$6.1 \times 10^{7}$ cells

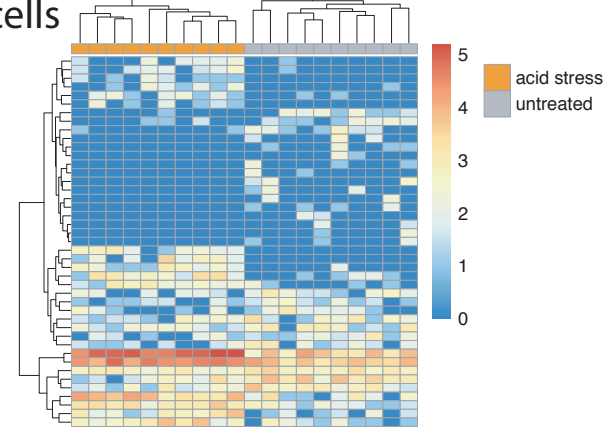

$5.3 \times 10^{7}$ cells

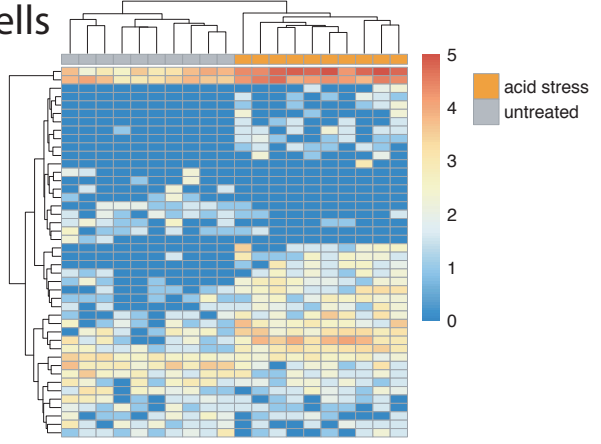

$4.4 \times 10^{7}$ cells

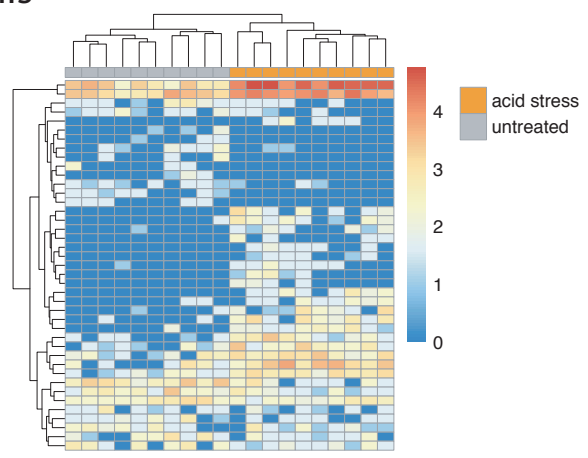

$3.5 \times 10^{7}$ cells

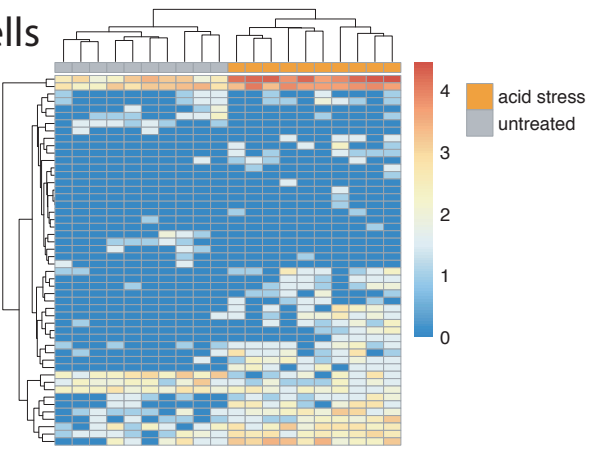

$2.6 \times 10^{7}$ cells

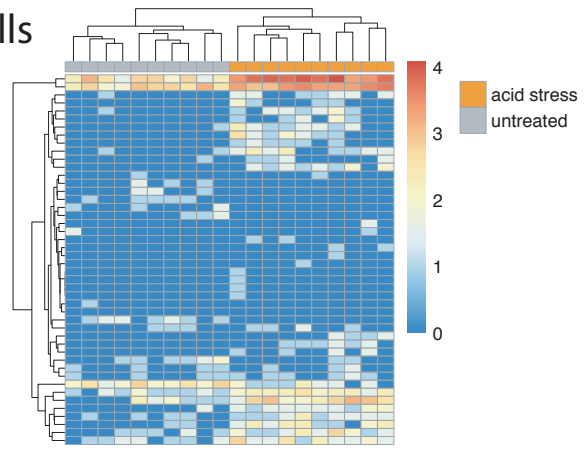

$1.8 \times 10^{7}$ cells

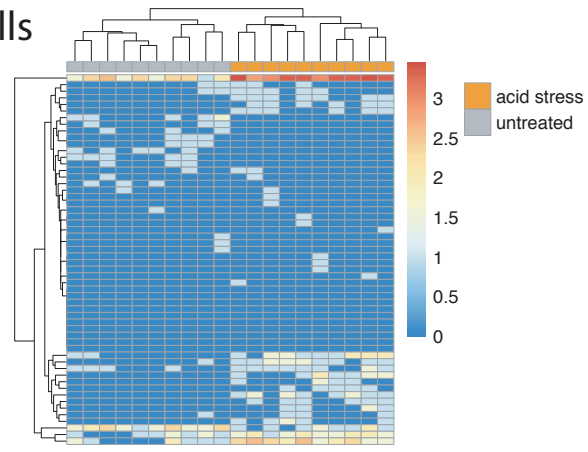

$8.8 \times 10^{6}$ cells

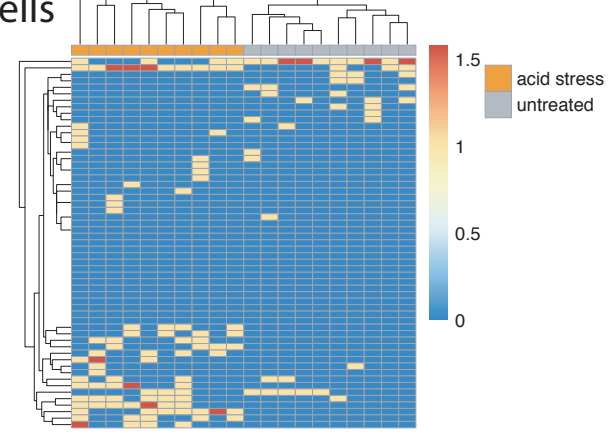




\section{Extended Data Figure 9 | Defining the minimum number of cells required for assessing complex cellular behaviours using Record-seq and differential expressed signature gene}

analysis. Using the acid stress response data set shown in Fig. 4e-g, differential expressed signature genes were identified for the entire data set as well as progressively and randomly downsampled data. The plots depict hierarchically clustered signature gene heatmaps. These data show that with $10 \%$ of the original data (corresponding to 448 spacer or $8.8 \times 10^{6}$ E. coli cells) the signature genes can appropriately classify the samples. The calculation of cell numbers is described in detail in Supplementary Notes; $n=10$ independent biological samples. 


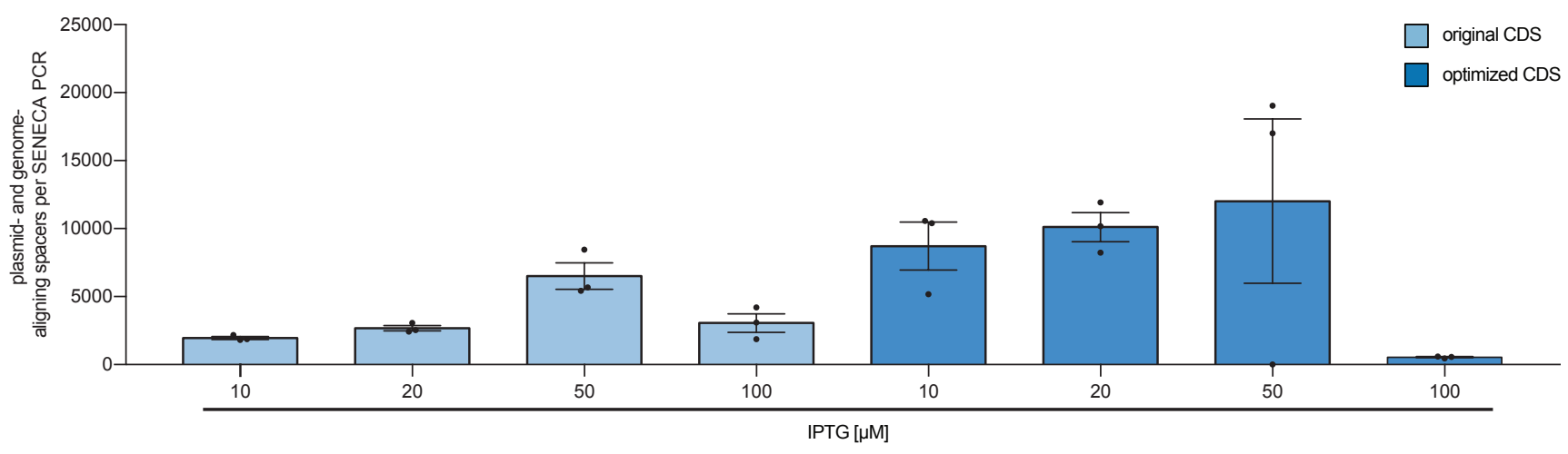

b

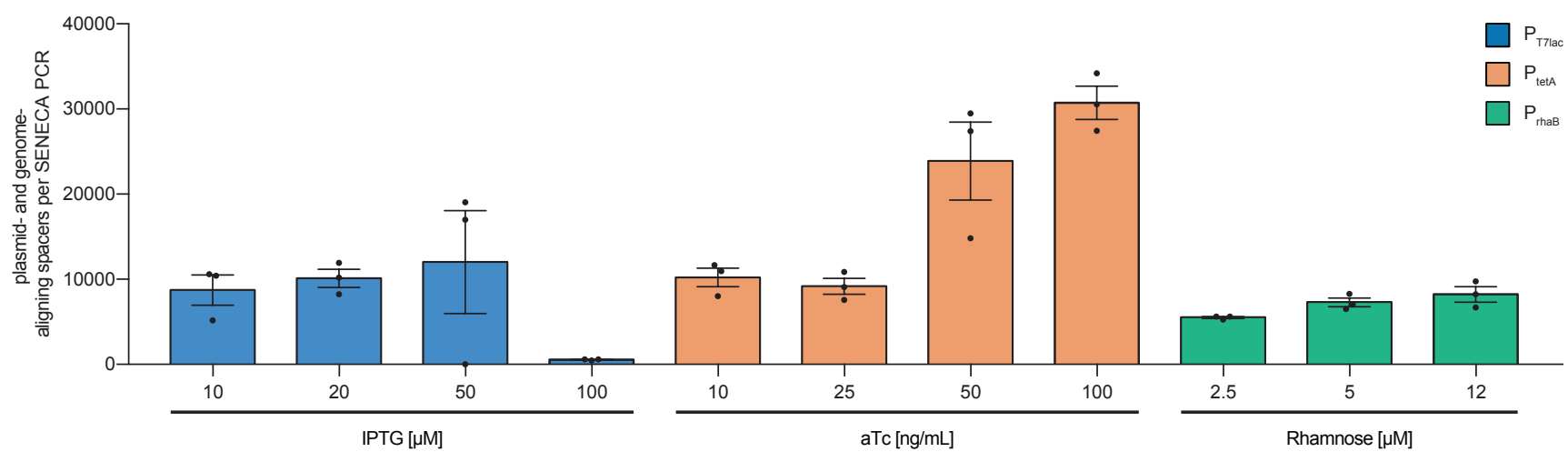

RNA-seq

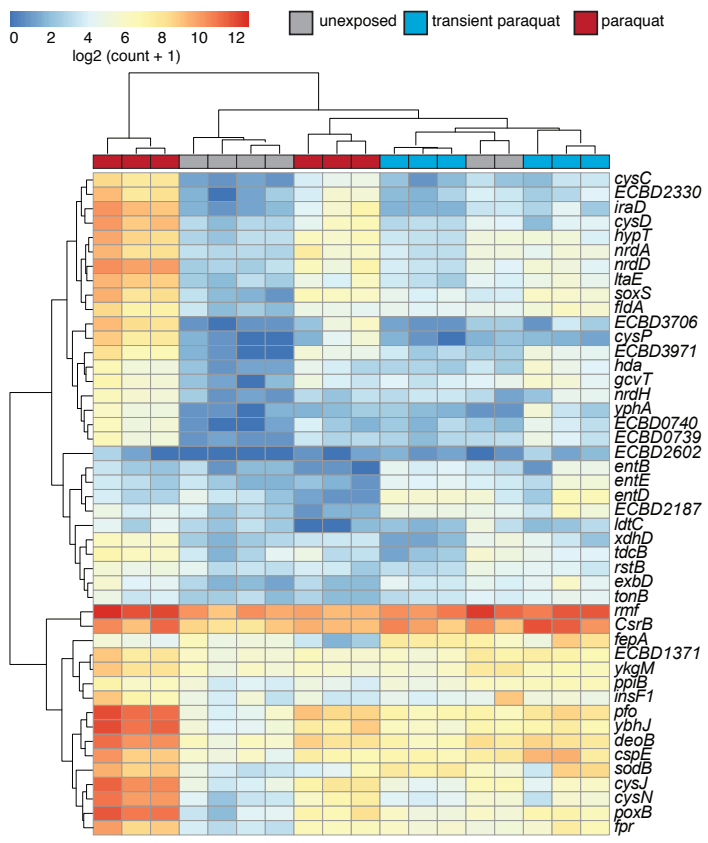

d

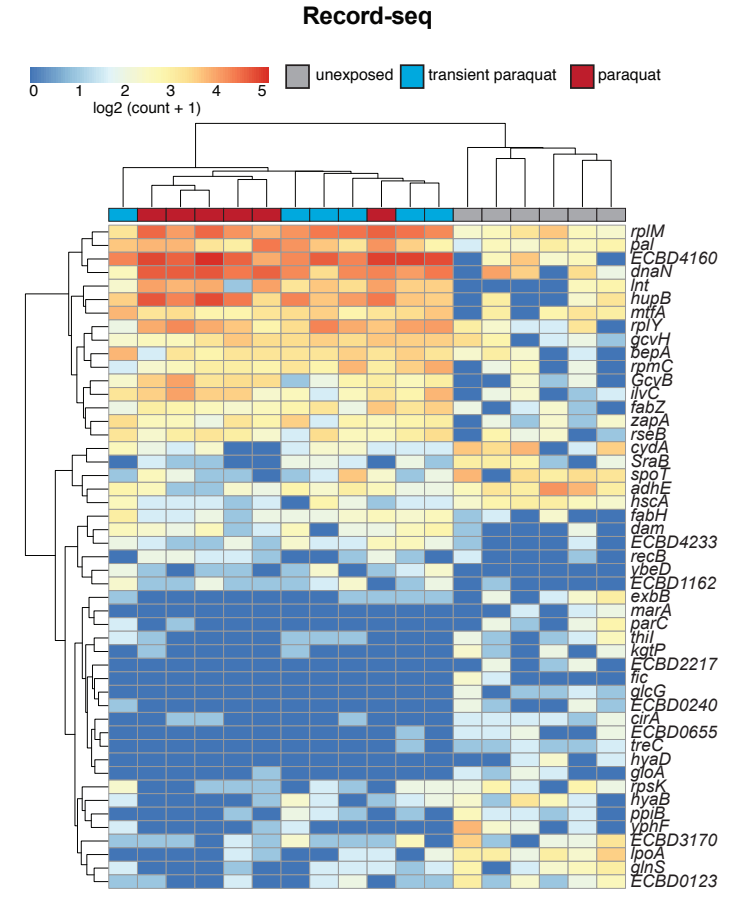




\section{Extended Data Figure 10 | Optimization of CRISPR spacer acquisition efficiency and}

detection of signature genes corresponding to Record-seq-compatible sentinel cells for encoding transient herbicide exposure. a, Plasmid and genome-aligning spacers obtained from E. coli BL21(DE3) transformed with FsRT-Cas1-Cas2 encoding plasmid using the original coding sequence (CDS) (light blue) or optimized CDS (dark blue) under the indicated IPTG concentrations. b, Plasmid and genome-aligning spacers obtained from E.coli BL21(DE3) transformed with $F_{S} \mathrm{RT}$-Cas1-Cas2 encoding plasmid using the optimized coding sequence under transcriptional control of the $\mathrm{P}_{\mathrm{T} 7 \mathrm{lac}}, \mathrm{P}_{\text {tet }}$, or $\mathrm{P}_{\text {rhaB }}$ promoter, induced with the indicated concentrations of IPTG, aTc, or Rhamnose, respectively. c, Unsupervised hierarchical clustering of RNA-seq cumulative expression profiles for signature differentially (cumulatively) expressed genes. Signature genes represent the union between the top 20 most differently expressed genes identified by DESeq2, edgeR, and baySeq, $n=6$ independent biological samples. d, Unsupervised hierarchical clustering of Record-seq cumulative expression profiles for signature differentially (cumulatively) expressed genes. Signature genes represent the union between the top 20 most differently expressed genes identified by DESeq2, edgeR, and baySeq, $n=6$ independent biological samples. Data in $\mathbf{a}, \mathbf{b}$ are mean \pm s.e.m., $n=3$ independent biological samples. 